\title{
On the Rate of Convergence of Some New Modified Iterative Schemes
}

\author{
Renu Chugh, Sanjay Kumar* \\ Department of Mathematics, M.D.U, Rohtak, India \\ Email: *sanjay.sanju84@gmail.com
}

Received September 2, 2013; revised October 2, 2013; accepted October 9, 2013

Copyright (c) 2013 Renu Chugh, Sanjay Kumar. This is an open access article distributed under the Creative Commons Attribution License, which permits unrestricted use, distribution, and reproduction in any medium, provided the original work is properly cited.

\begin{abstract}
In this article, following Bizare and Amriteimoori [1] and B. Parsad and R. Sahni [2], we modify Ishikawa, Agarwal et al., Noor, SP iterative schemes and compare the rate of convergence of Ishikawa, Agarwal et al., Noor, SP and new modified Ishikawa, Agarwal et al., Noor, SP iterative schemes not only for particular fixed value of $\alpha_{n}, \beta_{n}, \gamma_{n}$ but also for varying the value of $\alpha_{n}, \beta_{n}, \gamma_{n}$. With the help of two numerical examples, we compare the converging step.
\end{abstract}

Keywords: Metric Space; New Modified Ishikawa; New Modified Agarwal et al.; New Modified SP; New Modified Noor

\section{Introduction}

Let $X$ be a complete metric space and $T$ be self map, then $F_{T}=\{x \in X, T x=x\}$ is called set of fixed points of $T$. Now in literature, there are several iteration processes to find the fixed point of any equation. In complete metric space, Picard iteration process is defined as

$$
x_{n+1}=T\left(x_{n}\right), n=0,1, \cdots
$$

which is used to approximate fixed points of mappings satisfying the condition

$$
d(T x, T y) \leq a d(x, y) \text {, where } 0 \leq a<1
$$

called Banach contraction condition.

In Ishikawa iteration process [3], $\left\{x_{n}\right\}_{n=0}^{\infty}$ is defined as

$$
\begin{aligned}
& x_{n+1}=\left(1-\alpha_{n}\right) x_{n}+\alpha_{n} T y_{n} \\
& y_{n}=\left(1-\beta_{n}\right) x_{n}+\beta_{n} T x_{n}
\end{aligned}
$$

where $\alpha_{n}, \beta_{n}$ are real sequences in [0,1].

Now for Agarwal et al. iteration [4], $\left\{x_{n}\right\}_{n=0}^{\infty}$ is defined as

$$
\begin{aligned}
& x_{n+1}=\left(1-\alpha_{n}\right) T x_{n}+\alpha_{n} T y_{n} \\
& y_{n}=\left(1-\beta_{n}\right) x_{n}+\beta_{n} T x_{n}
\end{aligned}
$$

*Corresponding author. where $\alpha_{n}, \beta_{n}$ are real sequences in [0,1].

M. A. Noor defines [5] $\left\{x_{n}\right\}_{n=0}^{\infty}$ as

$$
\begin{aligned}
& x_{n+1}=\left(1-\alpha_{n}\right) x_{n}+\alpha_{n} T y_{n} \\
& y_{n}=\left(1-\beta_{n}\right) x_{n}+\beta_{n} T z_{n} \\
& z_{n}=\left(1-\gamma_{n}\right) x_{n}+\gamma_{n} T x_{n}
\end{aligned}
$$

where $\alpha_{n}, \beta_{n}, \gamma_{n}$ are real sequences in $[0,1]$.

For SP iteration [6] $\left\{x_{n}\right\}_{n=0}^{\infty}$ is defined as

$$
\begin{aligned}
& x_{n+1}=\left(1-\alpha_{n}\right) y_{n}+\alpha_{n} T y_{n} \\
& y_{n}=\left(1-\beta_{n}\right) z_{n}+\beta_{n} T z_{n} \\
& z_{n}=\left(1-\gamma_{n}\right) x_{n}+\gamma_{n} T x_{n}
\end{aligned}
$$

where $\alpha_{n}, \beta_{n}, \gamma_{n}$ are real sequences in $[0,1]$.

\section{Preliminaries}

In this paper following Bizare and Amriteimoori [1] and B. Parsad and R. Sahni [2] we prove the basic results in sequel. In [1] Bizare and Amriteimoori improved the picard iteration under following conditions:

1) Initial approximation is chosen in the interval $[a, b]$, where function is defined.

2) Function has continuous derivative on $(a, b)$.

3) $\left|T^{\prime}(x)\right|<1$ for all $x \in[a, b]$

4) $a \leq T(x) \leq b$ for all $x \in[a, b]$ 
Definition 2.1 [1]. Let $\left\{x_{n}\right\}$ converges to $\alpha$. If there exists an integer constant $q$ and a real +ve constant $C$ such that

$$
\lim _{n \rightarrow \infty}\left|\frac{x_{n+1}-\alpha}{\left(x_{n}-\alpha\right)^{q}}\right|=C
$$

$q$ is called order and $C$ is called constant of convergence

Theorem 2.2([1,7]). Let $f \in C^{q}[a, b]$, if $f^{k}(x)=0$ for $k=1,2, \cdots, q-1$ and $f^{q}(x) \neq 0$ then sequence $\left\{x_{n}\right\}$ is of order $q$.

To improve the order of convergence of fixed iterative schemes, such that

$f^{\prime}(\alpha), f^{\prime \prime}(\alpha), \cdots, f^{k}(\alpha)=0$. We determines

$\lambda_{i}(i=1,2, \cdots, k)$ from the following equation

$$
\begin{aligned}
& x+\lambda_{1} x+\lambda_{2} x^{2}+\cdots+\lambda_{k} x^{k} \\
& =f(x)+\lambda_{1} x+\lambda_{2} x^{2}+\cdots+\lambda_{k} x^{k}
\end{aligned}
$$

which becomes

$x=\frac{f(x)+\lambda_{1} x+\lambda_{2} x^{2}+\cdots+\lambda_{k} x^{k}}{1+\lambda_{1}+\lambda_{2} x+\cdots+\lambda_{k} x^{k-1}}=f_{\lambda}(x)$ this is fixed point equation form .Now the assumption that $f_{\lambda}^{\prime}(\alpha)=f_{\lambda}^{\prime \prime}(\alpha)=\cdots=f_{\lambda}^{k-1}(\alpha)=0$ yields to a system of linear equations which after solving [1] converted into upper triangular matrix which have nonzero diagonal entries. It means determinant is nonzero. So we determine $\lambda_{i}(i=1,2, \cdots, k)$ uniquely.

Now the new Picard iteration becomes

$$
x_{n+1}=f_{\lambda}\left(x_{n}\right) n=1,2, \cdots .
$$

where

$$
f_{\lambda}(x)=\frac{f(x)+\lambda_{1} x+\lambda_{2} x^{2}+\cdots+\lambda_{k} x^{k}}{1+\lambda_{1}+\lambda_{2} x+\cdots+\lambda_{k} x^{k-1}}
$$

Following Bhagwati Parsad and Ritu Shani [2] the new modified Ishikawa, Agarwal et al., Noor, SP iterations are defined as:

New modified Ishikawa iteration scheme

$$
\begin{aligned}
& x_{n+1}=\left(1-\alpha_{n}\right) x_{n}+\alpha_{n} f_{\lambda}\left(y_{n}\right) \\
& \left.y_{n}=\left(1-\beta_{n}\right)\right) x_{n}+\beta_{n} f_{\lambda}\left(x_{n}\right)
\end{aligned}
$$

New modified Agarwal et al. iteration scheme

$$
\begin{aligned}
& x_{n+1}=\left(1-\alpha_{n}\right) f_{\lambda}\left(x_{n}\right)+\alpha_{n} f_{\lambda}\left(y_{n}\right) \\
& y_{n}=\left(1-\beta_{n}\right) x_{n}+\beta_{n} f_{\lambda}\left(x_{n}\right)
\end{aligned}
$$

New modified Noor iteration scheme

$$
\begin{aligned}
& x_{n+1}=\left(1-\alpha_{n}\right) x_{n}+\alpha_{n} f_{\lambda}\left(y_{n}\right) \\
& y_{n}=\left(1-\beta_{n}\right) x_{n}+\beta_{n} f_{\lambda}\left(z_{n}\right) \\
& z_{n}=\left(1-\gamma_{n}\right) x_{n}+\gamma_{n} f_{\lambda}\left(x_{n}\right)
\end{aligned}
$$

New modified SP iteration scheme

$$
\begin{aligned}
& x_{n+1}=\left(1-\alpha_{n}\right) y_{n}+\alpha_{n} f_{\lambda}\left(y_{n}\right) \\
& y_{n}=\left(1-\beta_{n}\right) z_{n}+\beta_{n} f_{\lambda}\left(z_{n}\right) \\
& z_{n}=\left(1-\gamma_{n}\right) x_{n}+\gamma_{n} f_{\lambda}\left(x_{n}\right)
\end{aligned}
$$

where $\left\{\alpha_{n}\right\},\left\{\beta_{n}\right\}$ and $\left\{\gamma_{n}\right\}$ are real sequences in [0, $1]$.

In this article, we compare the rate of convergence of new modified iterative schemes and simple iterative schemes with the help of the following examples

$$
\begin{aligned}
& p_{1}(x)=\mathrm{e}^{(1-x)^{2}}-1-x \\
& p_{2}(x)=\frac{3}{8}+\frac{35}{8} x^{4}-\frac{15}{4} x^{2}
\end{aligned}
$$

To find the fixed point we write $p_{1}(x)$ and $p_{2}(x)$ as

$$
\mathrm{e}^{(1-x)^{2}}-1-x=0 \text { and } \frac{3}{8}+\frac{35}{8} x^{4}-\frac{15}{4} x^{2}=0
$$

both equations has unique root in the interval $(0,1)$ so we convert this in the fixed point form

$$
x=\mathrm{e}^{(1-x)^{2}}-1=f(x) \text { and } x=\frac{\sqrt{90+1050 x^{4}}}{30}=f(x)
$$

and take $\alpha=0.5$ and $\alpha=0.35$ respectively. Now we solve it by

$$
f_{\lambda}(x)=\frac{f(x)+\lambda_{1} x+\lambda_{2} x^{2}+\cdots+\lambda_{k} x^{k}}{1+\lambda_{1}+\lambda_{2} x+\cdots+\lambda_{k} x^{k-1}}
$$

For respective value of $\alpha, \lambda_{1}, \lambda_{2}, \lambda_{3}, \cdots, \lambda_{k}$ can be determined uniquely from system of linear equations as in [5] for $\alpha=0.5$ and $f(x)=\mathrm{e}^{(1-x)^{2}}-1$ we have

$$
\begin{aligned}
& \left(\begin{array}{ccccc}
1 & \alpha & \alpha^{2} & \alpha^{3} & \alpha^{4} \\
0 & 1 & 2 \alpha & 3 \alpha^{2} & 4 \alpha^{3} \\
0 & 0 & 2 & 6 \alpha & 12 \alpha^{2} \\
0 & 0 & 0 & 6 & 24 \alpha \\
0 & 0 & 0 & 0 & 24
\end{array}\right)\left(\begin{array}{c}
\lambda_{1} \\
\lambda_{2} \\
\lambda_{3} \\
\lambda_{4} \\
\lambda_{5}
\end{array}\right) \\
& =\left(\begin{array}{c}
f_{\lambda}^{\prime}(\alpha) \\
f_{\lambda}^{(2)}(\alpha) \\
f_{\lambda}^{(3)}(\alpha) \\
f_{\lambda}^{(4)}(\alpha) \\
f_{\lambda}^{(5)}(\alpha)
\end{array}\right)=\left(\begin{array}{c}
1.28403 \\
-3.85208 \\
8.98818 \\
-32.1006 \\
104.006
\end{array}\right)
\end{aligned}
$$

After solving the system of linear equations we have $\lambda_{1}=6.0858, \lambda_{2}=-17757, \lambda_{3}=22.2698$, $\lambda_{4}=-14.0173, \lambda_{5}=4.3336$

for second polynomial equation where $\alpha=0.35$ and

$$
f(x)=\frac{\sqrt{90+1050 x^{4}}}{30}
$$


System of linear equations become

$$
\begin{aligned}
& \left(\begin{array}{ccccc}
1 & \alpha & \alpha^{2} & \alpha^{3} & \alpha^{4} \\
0 & 1 & 2 \alpha & 3 \alpha^{2} & 4 \alpha^{3} \\
0 & 0 & 2 & 6 \alpha & 12 \alpha^{2} \\
0 & 0 & 0 & 6 & 24 \alpha \\
0 & 0 & 0 & 0 & 24
\end{array}\right)\left(\begin{array}{l}
\lambda_{1} \\
\lambda_{2} \\
\lambda_{3} \\
\lambda_{4} \\
\lambda_{5}
\end{array}\right) \\
& =\left(\begin{array}{c}
-f_{\lambda}^{\prime}(\alpha) \\
-f_{\lambda}^{(2)}(\alpha) \\
-f_{\lambda}^{(3)}(\alpha) \\
-f_{\lambda}^{(4)}(\alpha) \\
-f_{\lambda}^{(5)}(\alpha)
\end{array}\right)=\left(\begin{array}{c}
-0.291842 \\
-2.25304 \\
-8.53984 \\
32.6662 \\
422.235
\end{array}\right)
\end{aligned}
$$

$$
\begin{aligned}
& \lambda_{1}=0.0129005, \lambda_{2}=-0.330016, \lambda_{3}=3.01516, \\
& \lambda_{4}=17.5910, \lambda_{5}=-19.186
\end{aligned}
$$

\section{Experiments}

Now using the value of $\lambda_{1}, \lambda_{2}, \lambda_{3}, \cdots, \lambda_{k}$ and iterative schemes we have following Tables 1-24, and Figures 1-16.

\begin{tabular}{|c|c|c|c|c|c|c|c|c|}
\hline & \multicolumn{2}{|c|}{$a=0.2, b=0.9999$} & \multicolumn{2}{|c|}{$a=0.2, b=0.9$} & \multicolumn{2}{|c|}{$a=0.3, b=0.6$} & \multicolumn{2}{|c|}{$a=0.2, b=0.2$} \\
\hline$N$ & $x_{n+1}$ & $x_{n+1}$ & $x_{n+1}$ & $T x_{n}$ & $x_{n+1}$ & $T x_{n}$ & $x_{n+1}$ & $T x_{n}$ \\
\hline 0 & 0.0804248 & 0.0100502 & 0.162309 & 0.0100502 & 0.446501 & 0.0100502 & 0.736069 & 0.0100502 \\
\hline 1 & 0.56607 & 1.32942 & 0.519967 & 1.01723 & 0.41845 & 0.358473 & 0.622944 & 0.0721434 \\
\hline 2 & 0.291442 & 0.207189 & 0.346672 & 0.259144 & 0.413572 & 0.402422 & 0.548051 & 0.152774 \\
\hline 7 & 0.443316 & 0.483717 & 0.417293 & 0.426179 & 0.412392 & 0.412388 & 0.427266 & 0.375276 \\
\hline 8 & 0.388159 & 0.363285 & 0.409449 & 0.404313 & 0.412391 & 0.412391 & 0.421969 & 0.388222 \\
\hline 9 & 0.431032 & 0.454046 & 0.414152 & 0.417296 & 0.412391 & 0.412391 & 0.418561 & 0.39671 \\
\hline--- & --- & --- & --- & --- & --- & --- & --- & --- \\
\hline 27 & 0.412589 & 0.412814 & 0.412391 & 0.412392 & & & 0.412393 & 0.412385 \\
\hline 28 & 0.412238 & 0.412063 & 0.412391 & 0.412391 & & & 0.412393 & 0.412387 \\
\hline 29 & 0.41251 & 0.412646 & 0.412391 & 0.412391 & & & 0.412392 & 0.412389 \\
\hline --- & ----- & --- & --- & --- & ---- & ---- & --- & ---- \\
\hline 31 & 0.412463 & 0.412545 & & & & & 0.412392 & 0.41239 \\
\hline 32 & 0.412335 & 0.412272 & & & & & 0.412391 & 0.412391 \\
\hline 33 & 0.412435 & 0.412484 & & & & & 0.412391 & 0.412391 \\
\hline 55 & 0.412391 & 0.412392 & & & & & & \\
\hline 56 & 0.412391 & 0.412391 & & & & & & \\
\hline 57 & 0.412391 & 0.412391 & & & & & & \\
\hline
\end{tabular}

We take $\alpha_{n}=a, \beta_{n}=b, \gamma_{n}=c$

Hence we get

Table 1. Simple Ishikawa for $p_{1}(x)$. 
Table 2. Modified Ishikawa for $p_{1}(x)$.

\begin{tabular}{|c|c|c|c|c|c|c|c|c|}
\hline \multirow[b]{2}{*}{$N$} & \multicolumn{2}{|c|}{$a=0.1$ to $0.9, b=0.99$} & \multicolumn{2}{|c|}{$a=0.1$ to $0.9, b=0.9$} & \multicolumn{2}{|c|}{$a=0.1$ to $0.9, b=0.6$} & \multicolumn{2}{|c|}{$a=0.1$ to $0.9, b=0.1$} \\
\hline & $x_{n+1}$ & $T x_{n}$ & $x_{n+1}$ & $T x_{n}$ & $x_{n+1}$ & $T x_{n}$ & $x_{n+1}$ & $T x_{n}$ \\
\hline 0 & 0.424618 & 0.472629 & 0.481638 & 0.472629 & 0.654425 & 0.472629 & 0.942404 & 0.472629 \\
\hline 1 & 0.412376 & 0.412344 & 0.416257 & 0.408094 & 0.488042 & 0.370271 & 0.886798 & 0.420223 \\
\hline 2 & 0.412391 & 0.412391 & 0.412795 & 0.412411 & 0.440202 & 0.407234 & 0.834615 & 0.384598 \\
\hline 3 & 0.412391 & 0.412391 & 0.412434 & 0.412395 & 0.423255 & 0.411826 & 0.786785 & 0.364432 \\
\hline 4 & 0.412391 & 0.412391 & 0.412396 & 0.412392 & 0.41673 & 0.412365 & 0.743706 & 0.356248 \\
\hline 5 & & & 0.412392 & 0.412391 & 0.414139 & 0.412411 & 0.705366 & 0.356001 \\
\hline 6 & & & 0.412391 & 0.412391 & 0.413097 & 0.412404 & 0.671492 & 0.360233 \\
\hline 7 & & & 0.412391 & 0.412391 & 0.412677 & 0.412397 & 0.641687 & 0.366495 \\
\hline ------ & & & & & ------------ & ------------ & & \\
\hline 14 & & & & & 0.412392 & 0.412391 & 0.512968 & 0.400961 \\
\hline 15 & & & & & 0.412391 & 0.412391 & 0.502184 & 0.403247 \\
\hline 16 & & & & & 0.412391 & 0.412391 & 0.492625 & 0.405092 \\
\hline 100 & & & & & & & 0.412403 & 0.412391 \\
\hline 134 & & & & & & & 0.412392 & 0.412391 \\
\hline 135 & & & & & & & 0.412391 & 0.412391 \\
\hline 136 & & & & & & & 0.412391 & 0.412391 \\
\hline
\end{tabular}

Table 3. Simple Agarwal et al. for $p_{1}(x)$.

\begin{tabular}{|c|c|c|c|c|c|c|c|c|}
\hline \multirow[b]{2}{*}{$N$} & \multicolumn{2}{|c|}{$a=0.2, b=0.9999$} & \multicolumn{2}{|c|}{$a=0.2, b=0.9$} & \multicolumn{2}{|c|}{$a=0.3, b=0.6$} & \multicolumn{2}{|c|}{$a=0.5, b=0.5$} \\
\hline & $x_{n+1}$ & $T x_{n}$ & $x_{n+1}$ & $T x_{n}$ & $x_{n+1}$ & $T x_{n}$ & $x_{n+1}$ & $T x_{n}$ \\
\hline 0 & 0.0803358 & 0.0100502 & 0.0733136 & 0.0100502 & 0.090521 & 0.0100502 & 0.177931 & 0.0100502 \\
\hline 1 & 0.566185 & 1.3298 & 0.644644 & 1.3602 & 0.727194 & 1.2868 & 0.583438 & 0.965599 \\
\hline 25 & 0.412721 & 0.413097 & 0.418074 & 0.423399 & 0.420618 & 0.428339 & 0.412391 & 0.412392 \\
\hline 26 & 0.412135 & 0.411843 & 0.407474 & 0.403035 & 0.405294 & 0.398897 & 0.412391 & 0.412391 \\
\hline 27 & 0.412591 & 0.412817 & 0.416645 & 0.42061 & 0.418546 & 0.424292 & 0.412391 & 0.412391 \\
\hline 55 & 0.412391 & 0.412392 & 0.412465 & 0.412533 & 0.412498 & 0.412596 & & \\
\hline 56 & 0.412391 & 0.412391 & 0.412327 & 0.412269 & 0.412299 & 0.412214 & & \\
\hline 95 & & & 0.412391 & 0.412392 & 0.412391 & 0.412392 & & \\
\hline 96 & & & 0.412391 & 0.412391 & 0.412391 & 0.412391 & & \\
\hline 97 & & & & & 0.412391 & 0.412392 & & \\
\hline 98 & & & & & 0.412391 & 0.412391 & & \\
\hline
\end{tabular}


Table 4. Modified Agarwal et al. for $p_{1}(x)$.

\begin{tabular}{ccccccccc}
\hline & \multicolumn{2}{c}{$a=0.9, b=0.99$} & & $a=0.1, b=0.9$ & & $a=0.3, b=0.6$ & \multicolumn{2}{c}{$a=0.9, b=0.1$} \\
\hline$N$ & $x_{n+1}$ & $T x_{n}$ & $x_{n+1}$ & $T x_{n}$ & $x_{n+1}$ & $T x_{n}$ & $x_{n+1}$ & $T x_{n}$ \\
\hline 0 & 0.408091 & 0.472629 & 0.428901 & 0.472629 & 0.408937 & 0.472629 & 0.465458 & 0.472629 \\
1 & 0.412381 & 0.412331 & 0.412289 & 0.412252 & 0.412356 & 0.412346 & 0.410188 & 0.409941 \\
2 & $\mathbf{0 . 4 1 2 3 9 1}$ & $\mathbf{0 . 4 1 2 3 9 1}$ & 0.41239 & 0.41239 & 0.412391 & 0.412391 & 0.412368 & 0.412365 \\
3 & $\mathbf{0 . 4 1 2 3 9 1}$ & $\mathbf{0 . 4 1 2 3 9 1}$ & $\mathbf{0 . 4 1 2 3 9 1}$ & $\mathbf{0 . 4 1 2 3 9 1}$ & $\mathbf{0 . 4 1 2 3 9 1}$ & $\mathbf{0 . 4 1 2 3 9 1}$ & $\mathbf{0 . 4 1 2 3 9 1}$ & $\mathbf{0 . 4 1 2 3 9 1}$ \\
$\mathbf{4}$ & $\mathbf{0 . 4 1 2 3 9 1}$ & $\mathbf{0 . 4 1 2 3 9 1}$ & $\mathbf{0 . 4 1 2 3 9 1}$ & $\mathbf{0 . 4 1 2 3 9 1}$ & $\mathbf{0 . 4 1 2 3 9 1}$ & $\mathbf{0 . 4 1 2 3 9 1}$ & $\mathbf{0 . 4 1 2 3 9 1}$ & $\mathbf{0 . 4 1 2 3 9 1}$ \\
\hline
\end{tabular}

Table 5. Simple SP for $p_{1}(x)$.

\begin{tabular}{|c|c|c|c|c|c|c|c|c|}
\hline \multirow[b]{2}{*}{$N$} & \multicolumn{2}{|c|}{$a=0.1, b=0.1, c=0.999$} & \multicolumn{2}{|c|}{$a=0.1, b=0.1, c=0.9$} & \multicolumn{2}{|c|}{$a=0.5, b=0.5, c=0.5$} & \multicolumn{2}{|c|}{$a=0.1, b=0.1, c=0.1$} \\
\hline & $x_{n+1}$ & $T x_{n}$ & $x_{n+1}$ & $T x_{n}$ & $x_{n+1}$ & $T x_{n}$ & $x_{n+1}$ & $T x_{n}$ \\
\hline 0 & 0.0736477 & 0.0100502 & 0.139578 & 0.0100502 & 0.416514 & 0.0100502 & 0.667545 & 0.0100502 \\
\hline 1 & 0.701496 & 1.35874 & 0.616499 & 1.09662 & 0.412245 & 0.405588 & 0.531266 & 0.116866 \\
\hline 2 & 0.182726 & 0.0931953 & 0.271814 & 0.158438 & 0.412396 & 0.412634 & 0.463428 & 0.245717 \\
\hline 3 & 0.617407 & 0.950211 & 0.519974 & 0.699365 & 0.412391 & 0.412382 & 0.433328 & 0.333637 \\
\hline 4 & 0.242048 & 0.157633 & 0.334615 & 0.259135 & 0.412391 & 0.412391 & 0.420799 & 0.378667 \\
\hline 5 & 0.566158 & 0.776228 & 0.471698 & 0.556962 & 0.412391 & 0.412391 & 0.415737 & 0.398604 \\
\hline--- & ----- & & --- & --- & & & ---- & ----- \\
\hline 16 & 0.371841 & 0.341091 & 0.409891 & 0.406887 & & & 0.412391 & 0.412391 \\
\hline 17 & 0.448911 & 0.483766 & 0.41427 & 0.416556 & & & 0.412391 & 0.412391 \\
\hline--- & ---- & ---- & --- & ----- & --- & --- & --- & --- \\
\hline 50 & 0.41149 & 0.41072 & 0.412391 & 0.412391 & & & & \\
\hline 51 & 0.413197 & 0.413889 & 0.412391 & 0.412391 & & & & \\
\hline--- & ----- & ----- & ----- & ----- & ---- & --- & --- & --- \\
\hline 126 & 0.412391 & 0.412391 & & & & & & \\
\hline
\end{tabular}

Table 6. Modified SP for $p_{1}(x)$.

\begin{tabular}{|c|c|c|c|c|c|c|c|c|}
\hline & \multicolumn{2}{|c|}{$a=0.9, b=0.9, c=0.9999$} & \multicolumn{2}{|c|}{$a=0.9, b=0.9, c=0.9$} & \multicolumn{2}{|c|}{$a=0.2, b=0.9, c=0.1$} & \multicolumn{2}{|c|}{$a=0.1, b=0.1, c=0.1$} \\
\hline$N$ & $x_{n+1}$ & $T x_{n}$ & $x_{n+1}$ & $T x_{n}$ & $x_{n+1}$ & $T x_{n}$ & $x_{n+1}$ & $T x_{n}$ \\
\hline 0 & 0.412399 & 0.472629 & 0.412496 & 0.472629 & 0.436509 & 0.472629 & 0.844335 & 0.472629 \\
\hline 1 & 0.412391 & 0.412391 & 0.412391 & 0.412392 & 0.413946 & 0.411994 & 0.712973 & 0.367256 \\
\hline 2 & 0.412391 & 0.412391 & 0.412391 & 0.412391 & 0.412512 & 0.412403 & 0.618933 & 0.359118 \\
\hline 3 & 0.412391 & 0.412391 & 0.412391 & 0.412391 & 0.412401 & 0.412392 & 0.55555 & 0.37889 \\
\hline 4 & 0.412391 & 0.412391 & 0.412391 & 0.412391 & 0.412392 & 0.412391 & 0.512865 & 0.394474 \\
\hline 5 & & & & & 0.412391 & 0.412391 & 0.483669 & 0.403265 \\
\hline 6 & & & & & 0.412391 & 0.412391 & 0.463373 & 0.407829 \\
\hline ----- & ---------- & -------- & ---------- & ------- & ---------- & -------- & & \\
\hline 43 & & & & & & & 0.412392 & 0.412391 \\
\hline 44 & & & & & & & 0.412392 & 0.412391 \\
\hline 45 & & & & & & & 0.412391 & 0.412391 \\
\hline 46 & & & & & & & 0.412391 & 0.412391 \\
\hline
\end{tabular}


Table 7. Simple Noor for $p_{1}(x)$.

\begin{tabular}{|c|c|c|c|c|c|c|c|c|}
\hline \multirow[b]{2}{*}{$N$} & \multicolumn{2}{|c|}{$a=0.3, b=0.3, c=0.5$} & \multicolumn{2}{|c|}{$a=0.3, b=0.5, c=0.7$} & \multicolumn{2}{|c|}{$a=0.5, b=0.7, c=0.9$} & \multicolumn{2}{|c|}{$a=0.5, b=0.7, c=0.9$} \\
\hline & $x_{n+1}$ & $T x_{n}$ & $x_{n+1}$ & $T x_{n}$ & $x_{n+1}$ & $T x_{n}$ & $x_{n+1}$ & $T x_{n}$ \\
\hline 0 & 0.506337 & 0.0100502 & 0.449615 & 0.0100502 & 0.331926 & 0.0100502 & 0.381042 & 0.0100502 \\
\hline 1 & 0.417997 & 0.275966 & 0.410239 & 0.353812 & 0.506867 & 0.562555 & 0.442088 & 0.466838 \\
\hline 2 & 0.412427 & 0.40316 & 0.41258 & 0.415975 & 0.34732 & 0.275298 & 0.389512 & 0.365152 \\
\hline 3 & 0.412391 & 0.412331 & 0.412375 & 0.412077 & 0.485348 & 0.531107 & 0.433466 & 0.451642 \\
\hline 5 & 0.412391 & 0.412391 & 0.412391 & 0.412389 & 0.470593 & 0.508772 & 0.395663 & 0.378452 \\
\hline 6 & 0.412391 & 0.412391 & 0.412391 & 0.412391 & 0.367544 & 0.323489 & 0.427488 & 0.440836 \\
\hline 7 & & & 0.412391 & 0.412391 & 0.459703 & 0.491825 & 0.400159 & 0.38787 \\
\hline 75 & & & & & & & 0.412391 & 0.412392 \\
\hline 76 & & & & & & & 0.412391 & 0.412391 \\
\hline 155 & & & & & 0.412391 & 0.412392 & & \\
\hline 156 & & & & & 0.412391 & 0.412391 & & \\
\hline 157 & & & & & 0.412391 & 0.412391 & & \\
\hline
\end{tabular}

Table 8. Modified Noor for $p_{1}(x)$.

\begin{tabular}{|c|c|c|c|c|c|c|c|c|}
\hline \multirow[b]{2}{*}{$N$} & \multicolumn{2}{|c|}{$a=0.9, b=0.9, c=0.999$} & \multicolumn{2}{|c|}{$a=0.9, b=0.9, c=0.9$} & \multicolumn{2}{|c|}{$a=0.7, b=0.5, c=0.5$} & \multicolumn{2}{|c|}{$a=0.1, b=0.1, c=0.1$} \\
\hline & $x_{n+1}$ & $T x_{n}$ & $x_{n+1}$ & $T x_{n}$ & $x_{n+1}$ & $T x_{n}$ & $x_{n+1}$ & $T x_{n}$ \\
\hline 0 & 0.410381 & 0.472629 & 0.469342 & 0.472629 & 0.681606 & 0.472629 & 0.942022 & 0.472629 \\
\hline 1 & 0.412389 & 0.412368 & 0.418102 & 0.409542 & 0.539107 & 0.364431 & 0.886261 & 0.419927 \\
\hline 2 & 0.412391 & 0.412391 & 0.412967 & 0.412409 & 0.473981 & 0.398109 & 0.834065 & 0.384326 \\
\hline 3 & 0.412391 & 0.412391 & 0.412449 & 0.412396 & 0.442827 & 0.409028 & 0.78628 & 0.364285 \\
\hline 6 & & & 0.412391 & 0.412391 & 0.416194 & 0.4124 & 0.67112 & 0.360296 \\
\hline 7 & & & 0.412391 & 0.412391 & 0.4143 & 0.412411 & 0.641343 & 0.366574 \\
\hline 20 & & & & & 0.412391 & 0.412391 & 0.463812 & 0.409495 \\
\hline 21 & & & & & 0.412391 & 0.412391 & & \\
\hline 134 & & & & & & & 0.412392 & 0.412391 \\
\hline 135 & & & & & & & 0.412391 & 0.412391 \\
\hline
\end{tabular}




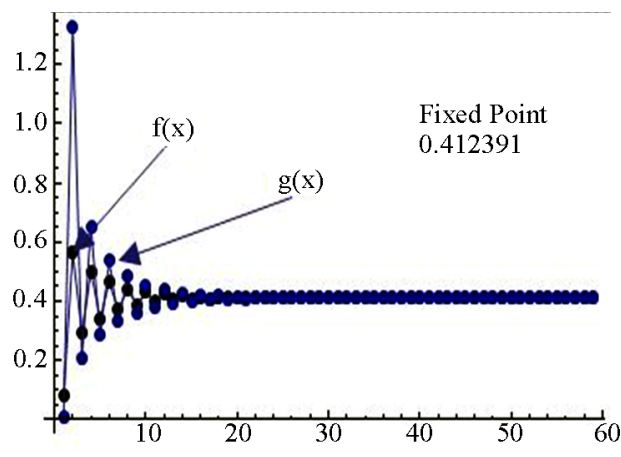

(a)

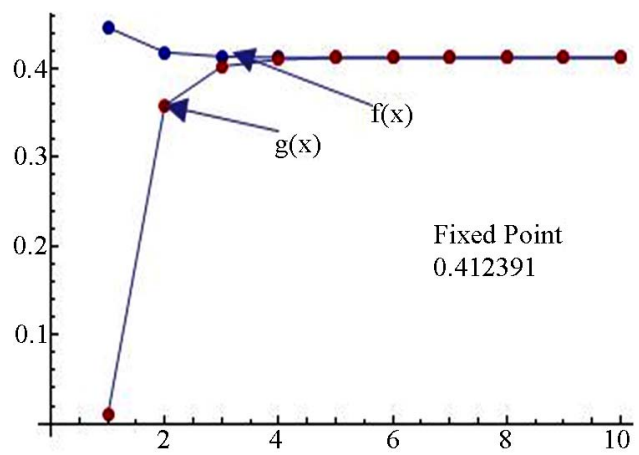

(c)

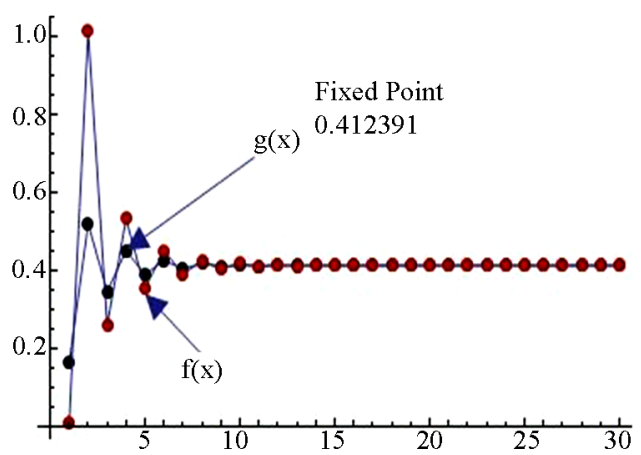

(b)

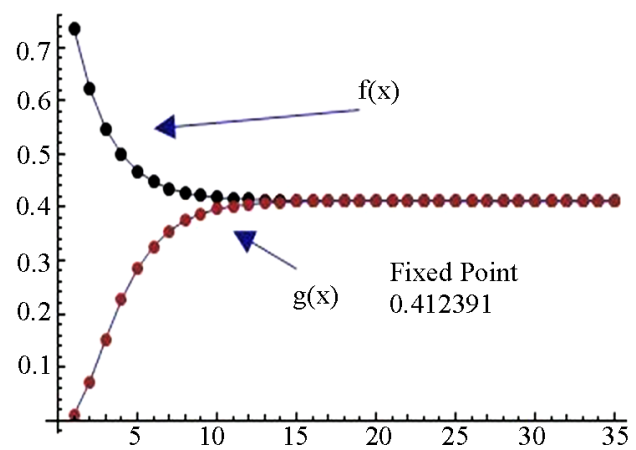

(d)

Figure 1. Graphical observations of simple Ishikawa iteration for $p_{1}(x)$. Here (a)-(d) show the graph for Table 1. The merging point with value 0.412391 is fixed point.

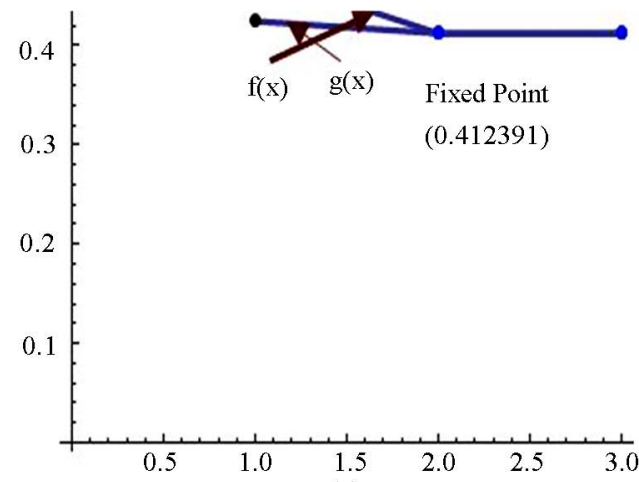

(a)

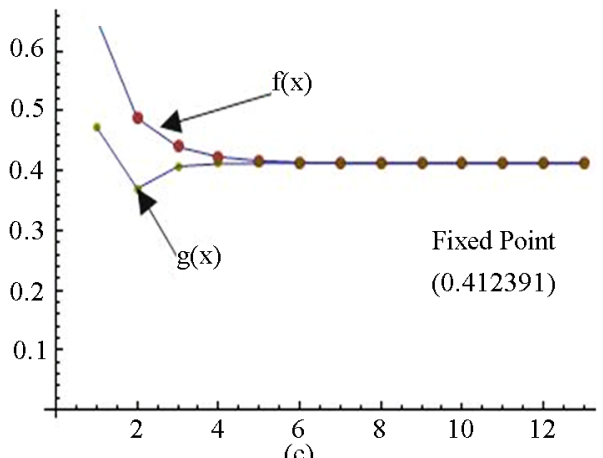

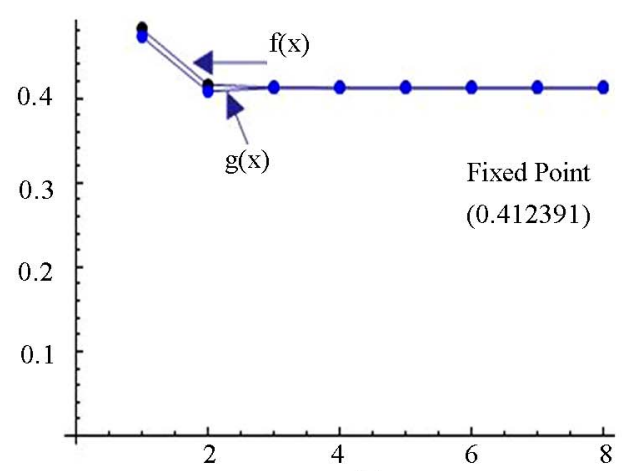

(b)

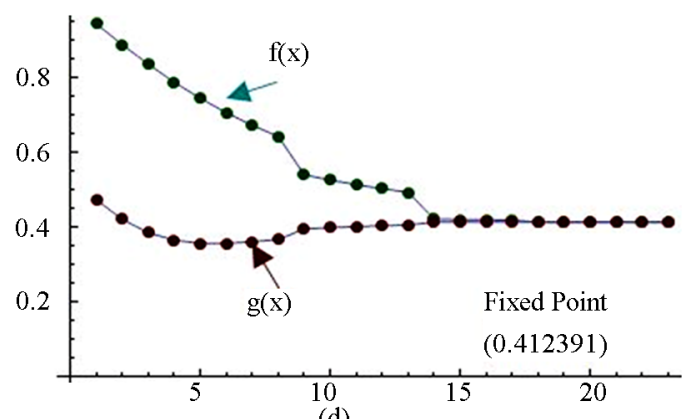

(d)

Figure 2. Graphical observations of new modified Ishikawa iteration for $p_{1}(x)$. Here (a)-(d) show the graph for Table 2. The merging point with value 0.412391 is fixed point. 


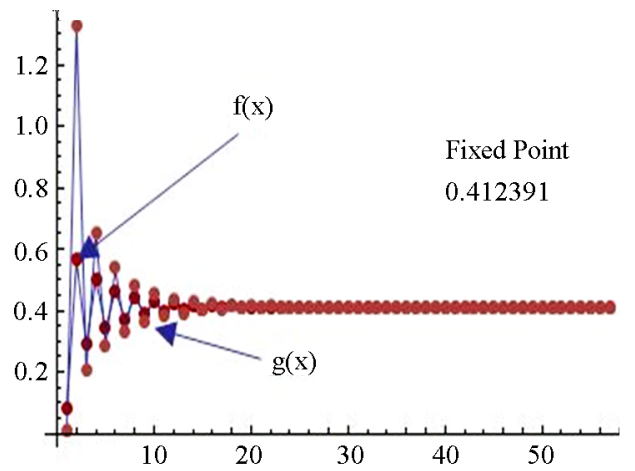

(a)

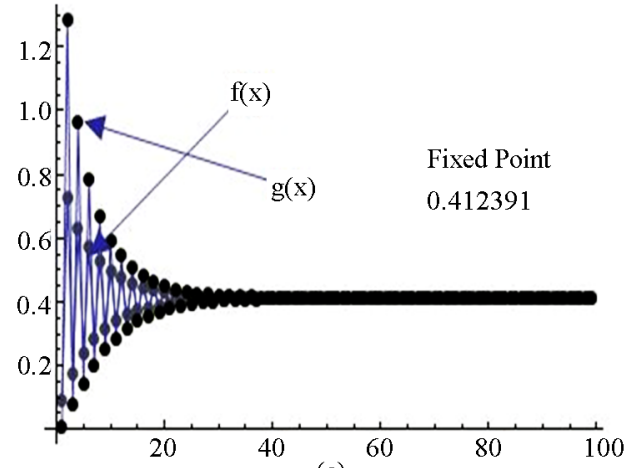

(c)

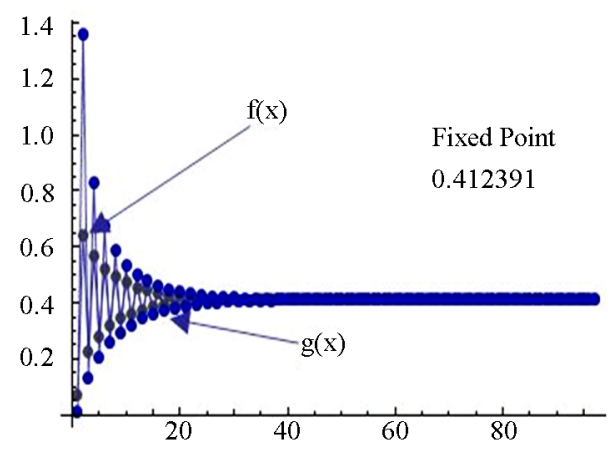

(b)

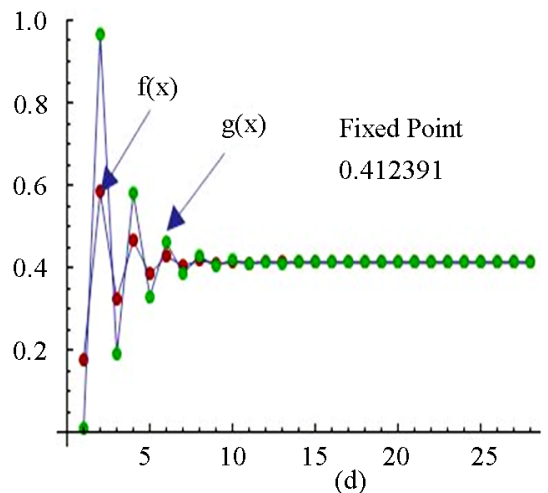

Figure 3. Graphical observations of simple Agarwal et al. iteration for $p_{1}(x)$. Here (a)-(d) show the graph for Table 3. The merging point with value 0.412391 is fixed point.

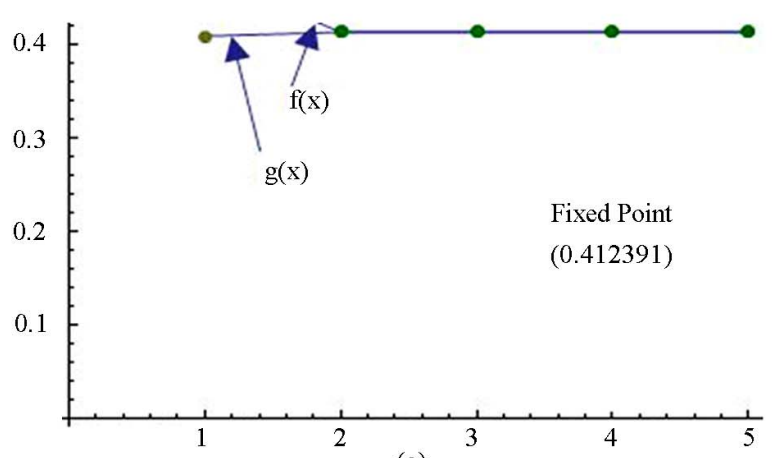

(a)

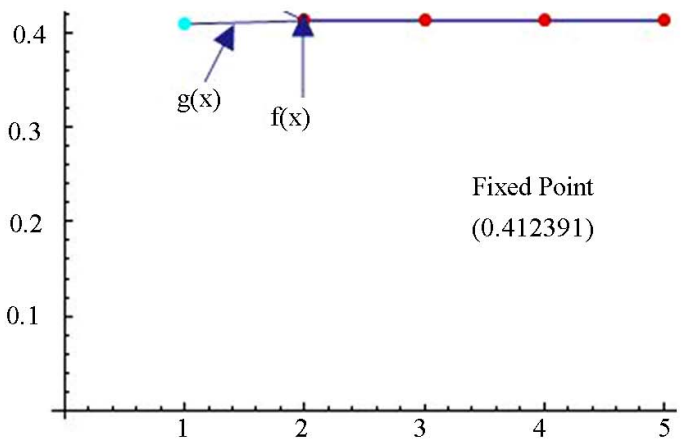

(c)

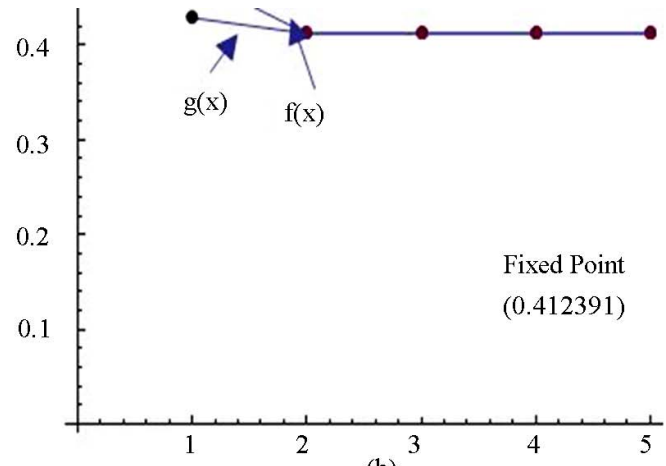

(b)

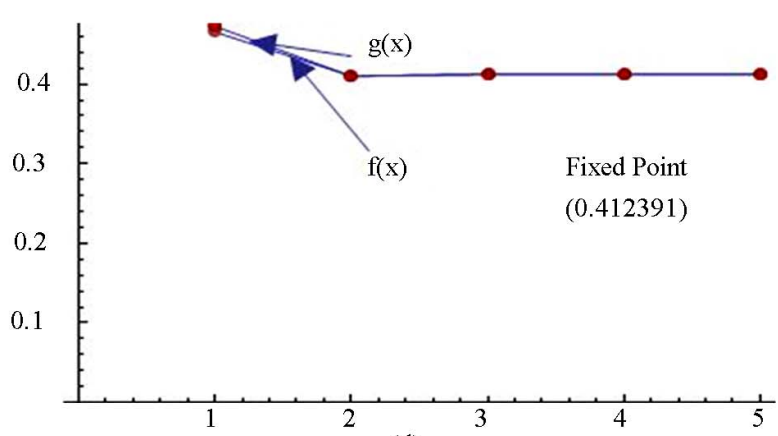

(d)

Figure 4. Graphical observations of new modified Agarwal et al. iteration for $p_{1}(x)$. Here (a)-(d) show the graph for Table 4. The merging point with value 0.412391 is fixed point. 


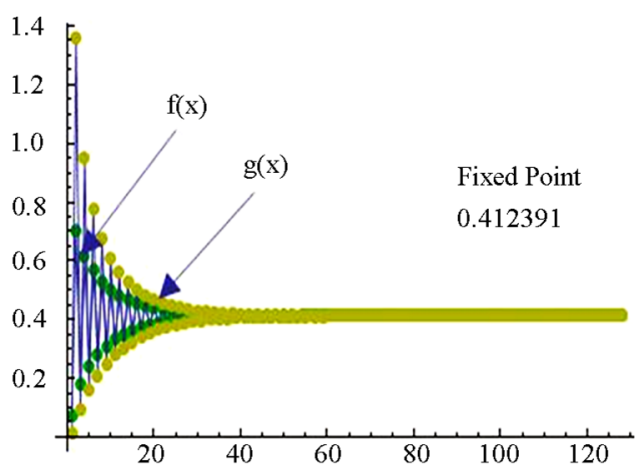

(a)

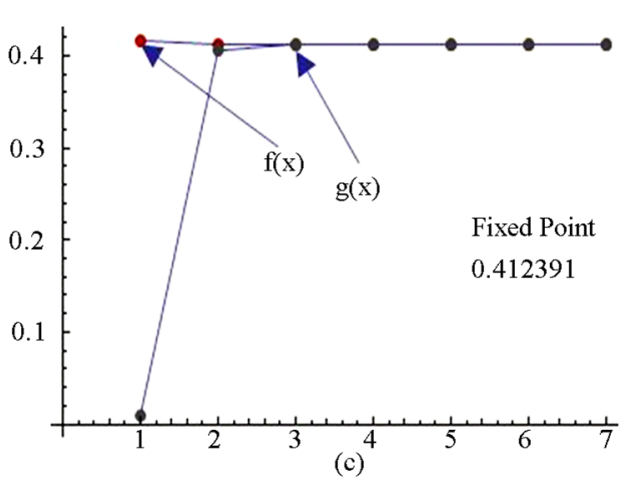

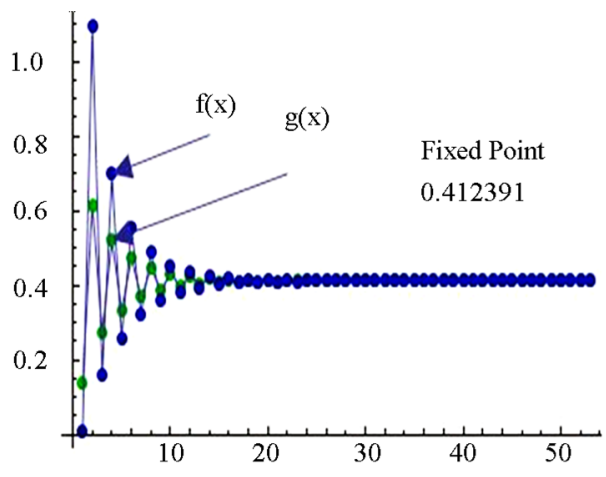

(b)

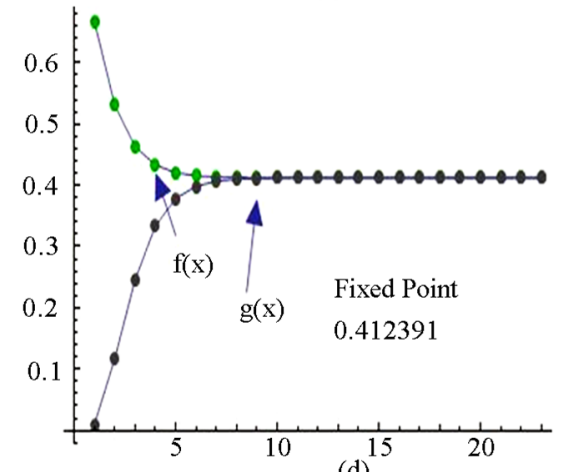

(d)

Figure 5. Graphical observations of simple SP iteration for $p_{1}(x)$. Here Here (a)-(d) show the graph for Table 5 . The merging point with value 0.412391 is fixed point.
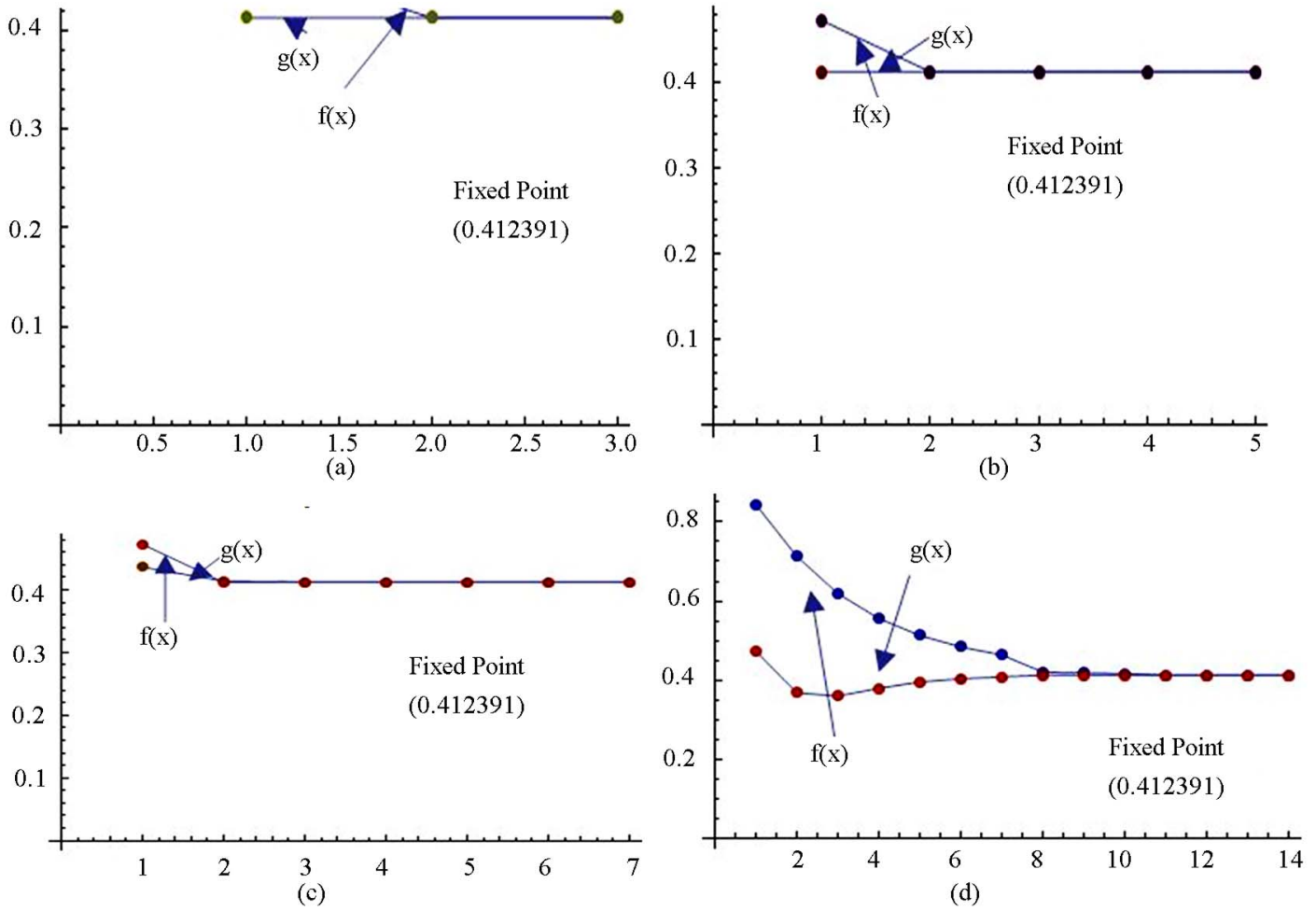

Figure 6. Graphical observations of new modified SP iteration for $p_{1}(x)$. Here (a)-(d) show the graph for Table 6. The merging point with value 0.412391 is fixed point. 


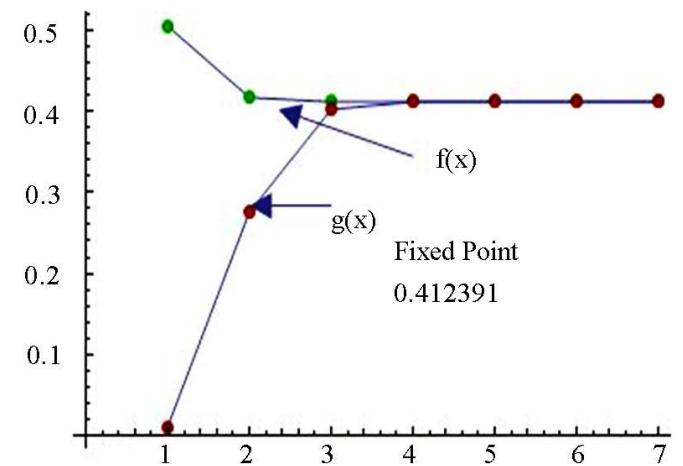

(a)

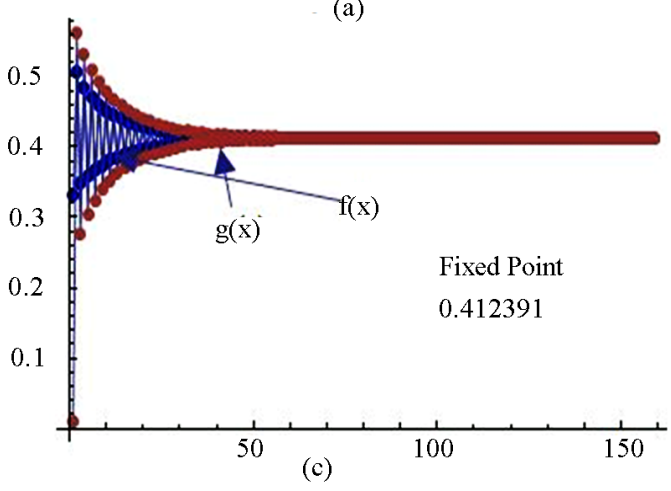

Figure 7. Graphical observations of simple Noor iteration for point with value 0.412391 is fixed point.

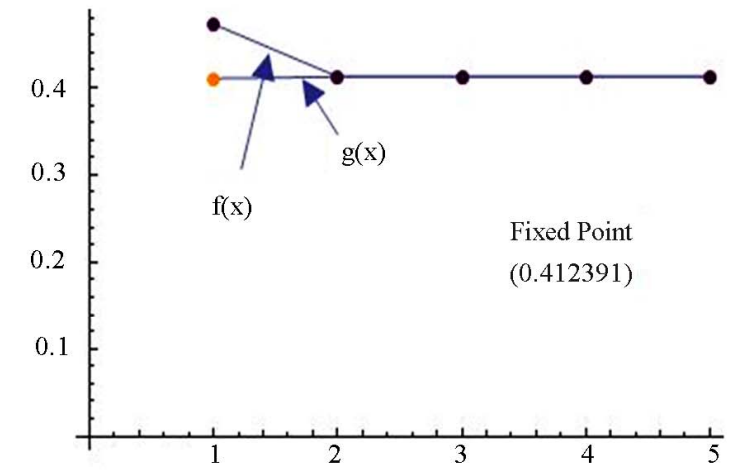

(a)

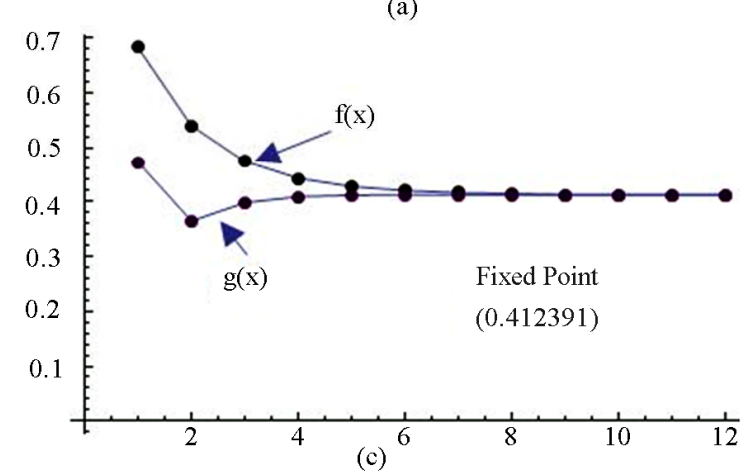

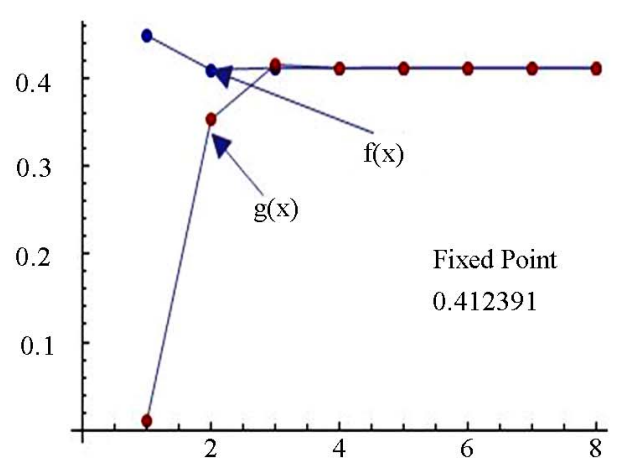

(b)

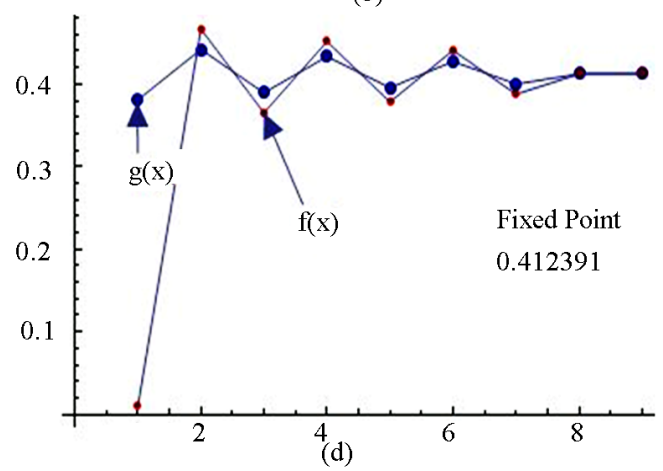

$p_{1}(x)$. Here (a)-(d) show the graph for Table 7. The merging

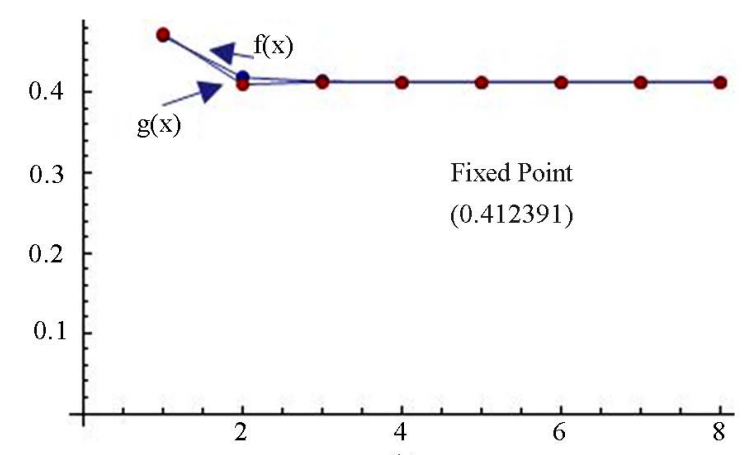

(b)

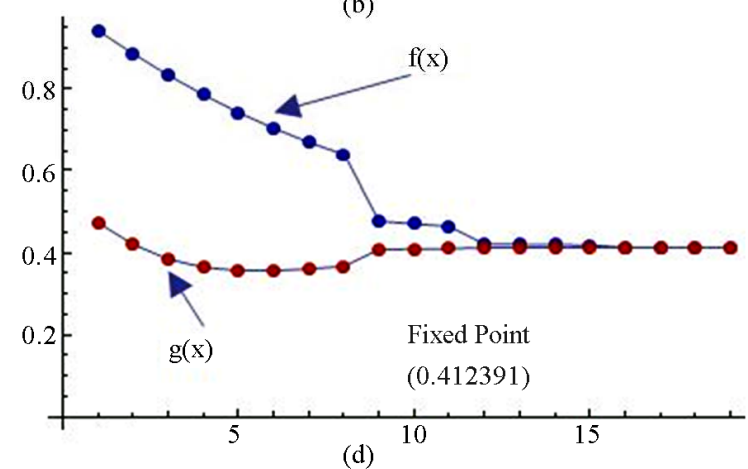

(d)

Figure 8. Graphical observations of new modified Noor iteration for $p_{1}(x)$. Here (a)-(d) show the graph for Table 8. The merging point with value 0.412391 is fixed point. 
Table 9. Simple Ishikawa for $p_{2}(x)$.

\begin{tabular}{cccccccccc}
\hline & \multicolumn{2}{c}{$a=0.9, b=0.999$} & \multicolumn{2}{c}{$a=0.1, b=0.9$} & \multicolumn{2}{c}{$a=0.3, b=0.5$} & \multicolumn{3}{c}{$a=0.1, b=0.1$} \\
\hline$N$ & $x_{n+1}$ & $T x_{n}$ & $x_{n+1}$ & $T x_{n}$ & $x_{n+1}$ & $T x_{n}$ & $x_{n+1}$ & $T x_{n}$ \\
\hline 0 & 0.340948 & 0.342793 & 0.343325 & 0.342793 & 0.346084 & 0.342793 & 0.349258 & 0.342793 \\
1 & 0.340071 & 0.340243 & 0.341078 & 0.340895 & 0.343689 & 0.341668 & 0.348571 & 0.342578 \\
2 & 0.339989 & 0.340005 & 0.340338 & 0.340278 & 0.342231 & 0.340996 & 0.347934 & 0.342379 \\
3 & 0.339982 & 0.339983 & 0.340097 & 0.340078 & 0.341345 & 0.340593 & 0.347344 & 0.342195 \\
6 & $\mathbf{0 . 3 3 9 9 8 1}$ & $\mathbf{0 . 3 3 9 9 8 1}$ & 0.339985 & 0.339984 & 0.340284 & 0.340116 & 0.345821 & 0.341726 \\
7 & $\mathbf{0 . 3 3 9 9 8 1}$ & $\mathbf{0 . 3 3 9 9 8 1}$ & 0.339982 & 0.339982 & 0.340164 & 0.340063 & 0.345386 & 0.341593 \\
9 & & & $\mathbf{0 . 3 3 9 9 8 1}$ & $\mathbf{0 . 3 3 9 9 8 1}$ & 0.340048 & 0.340011 & 0.344611 & 0.341357 \\
10 & & & $\mathbf{0 . 3 3 9 9 8 1}$ & $\mathbf{0 . 3 3 9 9 8 1}$ & 0.340022 & 0.339999 & 0.344266 & 0.341253 \\
18 & & & & 0.339982 & 0.339981 & 0.342284 & 0.340659 \\
20 & & & & $\mathbf{0 . 3 3 9 9 8 1}$ & $\mathbf{0 . 3 3 9 9 8 1}$ & 0.341952 & 0.340561 \\
21 & & & & $\mathbf{0 . 3 3 9 9 8 1}$ & $\mathbf{0 . 3 3 9 9 8 1}$ & 0.341805 & 0.340517 \\
127 & & & & & & 0.339982 & 0.339981 \\
128 & & & & & & & $\mathbf{0 . 3 3 9 9 8 1}$ & $\mathbf{0 . 3 3 9 9 8 1}$ \\
\hline
\end{tabular}

Table 10. Modified Ishikawa for $p_{2}(x)$.

\begin{tabular}{|c|c|c|c|c|c|c|c|c|}
\hline \multirow[b]{2}{*}{$N$} & \multicolumn{2}{|c|}{$a=0.9, b=0.9999$} & \multicolumn{2}{|c|}{$a=0.1, b=0.9$} & \multicolumn{2}{|c|}{$a=0.3, b=0.6$} & \multicolumn{2}{|c|}{$a=0.1, b=0.1$} \\
\hline & $x_{n+1}$ & $T x_{n}$ & $x_{n+1}$ & $T x_{n}$ & $x_{n+1}$ & $T x_{n}$ & $x_{n+1}$ & $T x_{n}$ \\
\hline 0 & 0.33998 & 0.339823 & 0.340869 & 0.339823 & 0.343944 & 0.339823 & 0.348985 & 0.339823 \\
\hline 1 & 0.339981 & 0.339981 & 0.340069 & 0.33998 & 0.341559 & 0.339957 & 0.348075 & 0.339854 \\
\hline 2 & 0.339981 & 0.339981 & 0.33999 & 0.339981 & 0.340611 & 0.339977 & 0.347257 & 0.339879 \\
\hline 4 & & & 0.339981 & 0.339981 & 0.340082 & 0.339981 & 0.345864 & 0.339915 \\
\hline 5 & & & 0.339981 & 0.339981 & 0.340021 & 0.339981 & 0.345271 & 0.339928 \\
\hline--- & & & & & ---------- & ----------- & & \\
\hline 9 & & & & & 0.339982 & 0.339981 & 0.343443 & 0.339959 \\
\hline 10 & & & & & 0.339981 & 0.339981 & 0.343096 & 0.339963 \\
\hline 11 & & & & & 0.339981 & 0.339981 & 0.342783 & 0.339966 \\
\hline 93 & & & & & & & 0.339982 & 0.339981 \\
\hline 94 & & & & & & & 0.339981 & 0.339981 \\
\hline 95 & & & & & & & 0.339981 & 0.339981 \\
\hline
\end{tabular}

Table 11. Simple Agarwal et al. for $p_{2}(x)$.

\begin{tabular}{ccccccccc}
\hline & \multicolumn{2}{c}{$a=0.9, b=0.99999}$, & \multicolumn{2}{c}{$a=0.1, b=0.9$} & \multicolumn{2}{c}{$a=0.3, b=0.5$} & \multicolumn{2}{c}{$a=0.1, b=0.1$} \\
\hline$N$ & $x_{n+1}$ & $T x_{n}$ & $x_{n+1}$ & $T x_{n}$ & $x_{n+1}$ & $T x_{n}$ & $x_{n+1}$ & $T x_{n}$ \\
\hline 0 & 0.340949 & 0.342793 & 0.342605 & 0.342793 & 0.342481 & 0.342793 & 0.342772 & 0.342793 \\
1 & 0.340071 & 0.340243 & 0.340649 & 0.340696 & 0.340587 & 0.340662 & 0.340737 & 0.340742 \\
2 & 0.339989 & 0.340005 & 0.34015 & 0.340162 & 0.340127 & 0.340145 & 0.340184 & 0.340185 \\
$\mathbf{5}$ & $\mathbf{0 . 3 3 9 9 8 1}$ & $\mathbf{0 . 3 3 9 9 8 1}$ & 0.339984 & 0.339984 & 0.339983 & 0.339983 & 0.339985 & 0.339985 \\
$\mathbf{6}$ & $\mathbf{0 . 3 3 9 9 8 1}$ & $\mathbf{0 . 3 3 9 9 8 1}$ & 0.339982 & 0.339982 & 0.339982 & 0.339982 & 0.339982 & 0.339982 \\
7 & $\mathbf{0 . 3 3 9 9 8 1}$ & $\mathbf{0 . 3 3 9 9 8 1}$ & $\mathbf{0 . 3 3 9 9 8 1}$ & $\mathbf{0 . 3 3 9 9 8 1}$ & $\mathbf{0 . 3 3 9 9 8 1}$ & $\mathbf{0 . 3 3 9 9 8 1}$ & $\mathbf{0 . 3 3 9 9 8 1}$ & $\mathbf{0 . 3 3 9 9 8 1}$ \\
8 & & & $\mathbf{0 . 3 3 9 9 8 1}$ & $\mathbf{0 . 3 3 9 9 8 1}$ & $\mathbf{0 . 3 3 9 9 8 1}$ & $\mathbf{0 . 3 3 9 9 8 1}$ & $\mathbf{0 . 3 3 9 9 8 1}$ & $\mathbf{0 . 3 3 9 9 8 1}$ \\
\hline
\end{tabular}


Table 12. Modified Aggarwal et al. for $p_{2}(x)$.

\begin{tabular}{cccccccccc}
\hline & \multicolumn{2}{c}{$a=0.99999, b=0.999$} & \multicolumn{2}{c}{$a=0.1, b=0.9$} & \multicolumn{2}{c}{$a=0.1, b=0.1$} & \multicolumn{2}{c}{$a=0.1, b=0.5$} \\
\hline$N$ & $x_{n+1}$ & $T x_{n}$ & $x_{n+1}$ & $T x_{n}$ & $x_{n+1}$ & $T x_{n}$ & $x_{n+1}$ & $T x_{n}$ \\
\hline 0 & 0.339981 & 0.339823 & 0.339851 & 0.339823 & 0.339827 & 0.339823 & 0.339839 & 0.339823 \\
1 & $\mathbf{0 . 3 3 9 9 8 1}$ & $\mathbf{0 . 3 3 9 9 8 1}$ & $\mathbf{0 . 3 3 9 9 8 1}$ & $\mathbf{0 . 3 3 9 9 8 1}$ & $\mathbf{0 . 3 3 9 9 8 1}$ & $\mathbf{0 . 3 3 9 9 8 1}$ & $\mathbf{0 . 3 3 9 9 8 1}$ & $\mathbf{0 . 3 3 9 9 8 1}$ \\
2 & $\mathbf{0 . 3 3 9 9 8 1}$ & $\mathbf{0 . 3 3 9 9 8 1}$ & $\mathbf{0 . 3 3 9 9 8 1}$ & $\mathbf{0 . 3 3 9 9 8 1}$ & $\mathbf{0 . 3 3 9 9 8 1}$ & $\mathbf{0 . 3 3 9 9 8 1}$ & $\mathbf{0 . 3 3 9 9 8 1}$ & $\mathbf{0 . 3 3 9 9 8 1}$ \\
\hline
\end{tabular}

Table 13. Simple SP for $p_{2}(x)$.

\begin{tabular}{|c|c|c|c|c|c|c|c|c|}
\hline \multirow[b]{2}{*}{$N$} & \multicolumn{2}{|c|}{$a=0.9, b=0.9, c=0.999$} & \multicolumn{2}{|c|}{$a=0.1, b=0.1, c=0.9$} & \multicolumn{2}{|c|}{$a=0.3, b=0.5, c=0.7$} & \multicolumn{2}{|c|}{$a=0.1, b=0.1, c=0.1$} \\
\hline & $x_{n+1}$ & $T x_{n}$ & $x_{n+1}$ & $T x_{n}$ & $x_{n+1}$ & $T x_{n}$ & $x_{n+1}$ & $T x_{n}$ \\
\hline 0 & 0.340313 & 0.342793 & 0.343012 & 0.342793 & 0.342455 & 0.342793 & 0.347988 & 0.342793 \\
\hline 1 & 0.339992 & 0.340071 & 0.340881 & 0.340808 & 0.340583 & 0.340655 & 0.346375 & 0.342211 \\
\hline 3 & 0.339981 & 0.339981 & 0.340059 & 0.340053 & 0.340016 & 0.34002 & 0.344053 & 0.341386 \\
\hline 4 & 0.339981 & 0.339981 & 0.340004 & 0.340002 & 0.33999 & 0.339991 & 0.343229 & 0.341097 \\
\hline-- & -- & ---------- & --------- & ---- & ----------- & ----------- & -------- & -------- \\
\hline 8 & & & 0.339981 & 0.339981 & 0.339981 & 0.339981 & 0.341292 & 0.340428 \\
\hline 9 & & & 0.339981 & 0.339981 & 0.339981 & 0.339981 & 0.341026 & 0.340337 \\
\hline -- & -- & ---------- & --------- & ---- & ------------ & ------------ & -------- & -------- \\
\hline 44 & & & & & & & 0.339981 & 0.339981 \\
\hline 45 & & & & & & & 0.339981 & 0.339981 \\
\hline
\end{tabular}

Table 14. Modified SP for $p_{2}(x)$.

\begin{tabular}{|c|c|c|c|c|c|c|c|c|}
\hline \multirow[b]{2}{*}{$N$} & \multicolumn{2}{|c|}{$a=0.9, b=0.9, c=0.999$} & \multicolumn{2}{|c|}{$a=0.1, b=0.1, c=0.9$} & \multicolumn{2}{|c|}{$a=0.3, b=0.5, c=0.7$} & \multicolumn{2}{|c|}{$a=0.1, b=0.1, c=0.1$} \\
\hline & $x_{n+1}$ & $T x_{n}$ & $x_{n+1}$ & $T x_{n}$ & $x_{n+1}$ & $T x_{n}$ & $x_{n+1}$ & $T x_{n}$ \\
\hline 0 & 0.340066 & 0.339823 & 0.34807 & 0.339823 & 0.343427 & 0.339823 & 0.34807 & 0.339823 \\
\hline 1 & 0.339982 & 0.339981 & 0.346515 & 0.339879 & 0.34118 & 0.339963 & 0.346515 & 0.339879 \\
\hline 2 & 0.339981 & 0.339981 & 0.345263 & 0.339915 & 0.3404 & 0.339979 & 0.345263 & 0.339915 \\
\hline 3 & 0.339981 & 0.339981 & 0.344252 & 0.339938 & 0.340128 & 0.339981 & 0.344252 & 0.339938 \\
\hline-- & -- & --------- & --------- & ---- & ----------- & ----------- & -------- & -------- \\
\hline 8 & & & 0.341463 & 0.339976 & 0.339982 & 0.339981 & 0.341463 & 0.339976 \\
\hline 9 & & & 0.341181 & 0.339978 & 0.339981 & 0.339981 & 0.341181 & 0.339978 \\
\hline 10 & & & 0.340953 & 0.339979 & 0.339981 & 0.339981 & 0.340953 & 0.339979 \\
\hline-- & -- & ----------- & --------- & ---- & ------------ & ------------ & -------- & ------- \\
\hline 46 & & & 0.339982 & 0.339981 & & & 0.339982 & 0.339981 \\
\hline 47 & & & 0.339981 & 0.339981 & & & 0.339981 & 0.339981 \\
\hline 48 & & & 0.339981 & 0.339981 & & & 0.339981 & 0.339981 \\
\hline
\end{tabular}


Table 15. Simple Noor for $p_{2}(x)$.

\begin{tabular}{|c|c|c|c|c|c|c|c|c|}
\hline \multirow[b]{2}{*}{$N$} & \multicolumn{2}{|c|}{$a=0.9, b=0.9, c=0.999$} & \multicolumn{2}{|c|}{$a=0.1, b=0.1, c=0.9$} & \multicolumn{2}{|c|}{$a=0.3, b=0.5, c=0.7$} & \multicolumn{2}{|c|}{$a=0.1, b=0.1, c=0.1$} \\
\hline & $x_{n+1}$ & $T x_{n}$ & $x_{n+1}$ & $T x_{n}$ & $x_{n+1}$ & $T x_{n}$ & $x_{n+1}$ & $T x_{n}$ \\
\hline 0 & 0.340491 & 0.342793 & 0.34332 & 0.342793 & 0.344161 & 0.342793 & 0.349258 & 0.342793 \\
\hline 1 & 0.340006 & 0.340119 & 0.341074 & 0.340894 & 0.341717 & 0.341127 & 0.34857 & 0.342577 \\
\hline 2 & 0.339982 & 0.339988 & 0.340337 & 0.340277 & 0.3407 & 0.340452 & 0.347932 & 0.342378 \\
\hline 3 & 0.339981 & 0.339981 & 0.340097 & 0.340077 & 0.340279 & 0.340176 & 0.347342 & 0.342195 \\
\hline 4 & 0.339981 & 0.339981 & 0.340019 & 0.340012 & 0.340104 & 0.340061 & 0.346794 & 0.342025 \\
\hline 5 & 0.339981 & 0.339981 & 0.339993 & 0.339991 & 0.340032 & 0.340014 & 0.346288 & 0.341869 \\
\hline 6 & & & 0.339985 & 0.339984 & 0.340002 & 0.339995 & 0.345818 & 0.341725 \\
\hline 7 & & & 0.339982 & 0.339982 & 0.33999 & 0.339987 & 0.345383 & 0.341592 \\
\hline 8 & & & 0.339981 & 0.339981 & 0.339985 & 0.339983 & 0.344981 & 0.34147 \\
\hline 9 & & & 0.339981 & 0.339981 & 0.339983 & 0.339982 & 0.344608 & 0.341357 \\
\hline 10 & & & & & 0.339982 & 0.339981 & 0.344263 & 0.341252 \\
\hline 11 & & & & & 0.339981 & 0.339981 & 0.343943 & 0.341156 \\
\hline 12 & & & & & 0.339981 & 0.339981 & 0.343647 & 0.341067 \\
\hline 13 & & & & & 0.339981 & 0.339981 & 0.343373 & 0.340984 \\
\hline-- & -- & ---------- & --------- & ---- & ------------ & ----------- & -------- & --------- \\
\hline 127 & & & & & & & 0.339982 & 0.339981 \\
\hline 128 & & & & & & & 0.339981 & 0.339981 \\
\hline 129 & & & & & & & 0.339981 & 0.339981 \\
\hline
\end{tabular}

Table 16. Modified Noor for $p_{2}(x)$.

\begin{tabular}{|c|c|c|c|c|c|c|c|c|}
\hline & \multicolumn{2}{|c|}{$a=0.9, b=0.9, c=0.999$} & \multicolumn{2}{|c|}{$a=0.1, b=0.1, c=0.9$} & \multicolumn{2}{|c|}{$a=0.3, b=0.5, c=0.7$} & \multicolumn{2}{|c|}{$a=0.1, b=0.1, c=0.1$} \\
\hline$N$ & $x_{n+1}$ & $T x_{n}$ & $x_{n+1}$ & $T x_{n}$ & $x_{n+1}$ & $T x_{n}$ & $x_{n+1}$ & $T x_{n}$ \\
\hline 0 & 0.340982 & 0.339823 & 0.348985 & 0.339823 & 0.344953 & 0.339823 & 0.348985 & 0.339823 \\
\hline 1 & 0.340081 & 0.33998 & 0.348075 & 0.339854 & 0.342458 & 0.339943 & 0.348075 & 0.339854 \\
\hline 2 & 0.339991 & 0.339981 & 0.347257 & 0.339879 & 0.341217 & 0.339972 & 0.347257 & 0.339879 \\
\hline 3 & 0.339982 & 0.339981 & 0.346523 & 0.339899 & 0.340599 & 0.339979 & 0.346523 & 0.339899 \\
\hline 4 & 0.339981 & 0.339981 & 0.345864 & 0.339915 & 0.34029 & 0.33998 & 0.345864 & 0.339915 \\
\hline 5 & 0.339981 & 0.339981 & 0.345271 & 0.339928 & 0.340135 & 0.339981 & 0.345271 & 0.339928 \\
\hline 13 & & & 0.342249 & 0.339972 & 0.339982 & 0.339981 & 0.342249 & 0.339972 \\
\hline 14 & & & 0.342022 & 0.339973 & 0.339981 & 0.339981 & 0.342022 & 0.339973 \\
\hline 15 & & & 0.341817 & 0.339975 & 0.339981 & 0.339981 & 0.341817 & 0.339975 \\
\hline 93 & & & 0.339982 & 0.339981 & & & 0.339982 & 0.339981 \\
\hline 94 & & & 0.339981 & 0.339981 & & & 0.339981 & 0.339981 \\
\hline
\end{tabular}




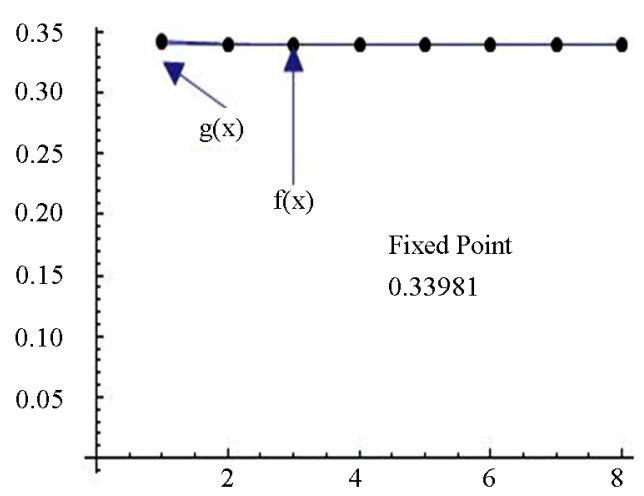

(a)

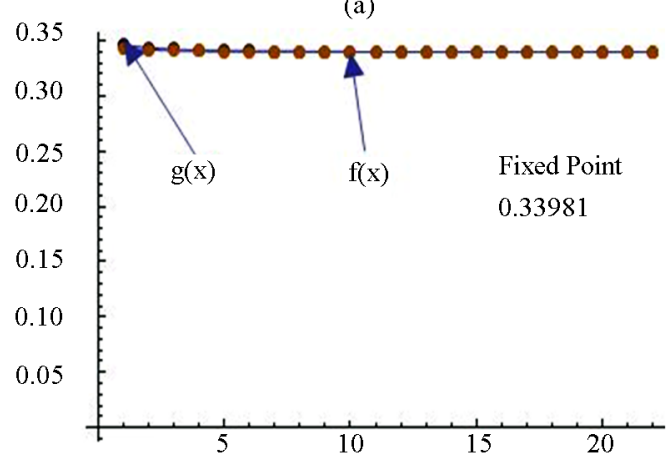

(c)

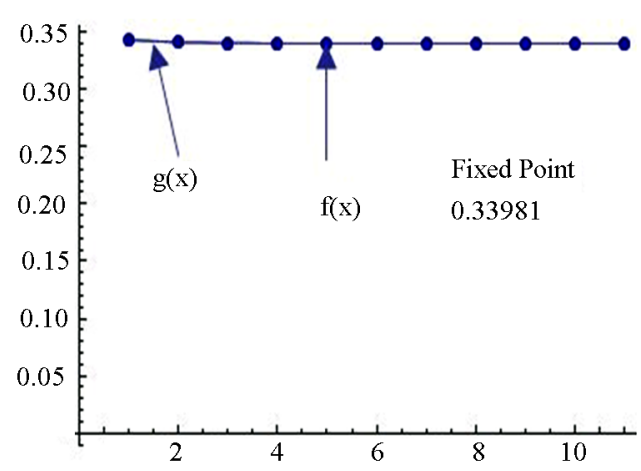

(b)

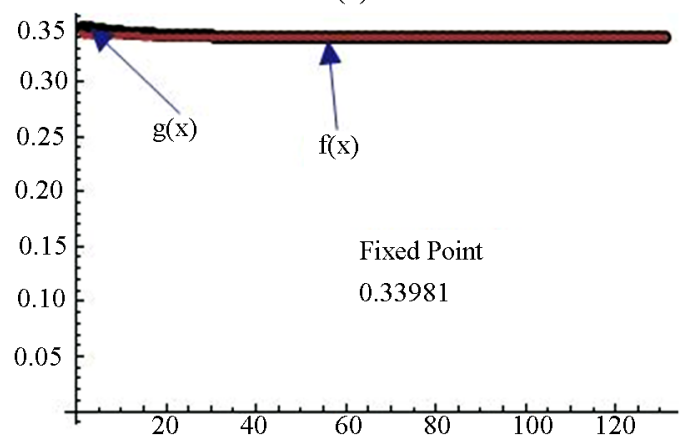

(d)

Figure 9. Graphical observations for simple Ishikawa iteration for $p_{2}(x)$. Here (a)-(d) show the graph for Table 9. The merging point with value 0.33981 is fixed point.
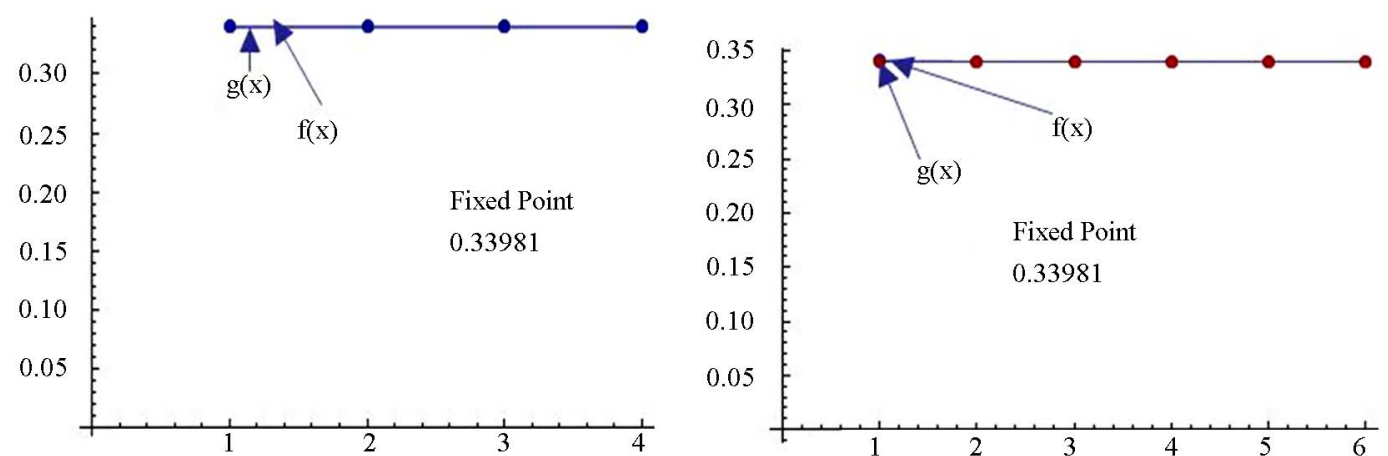

(a)

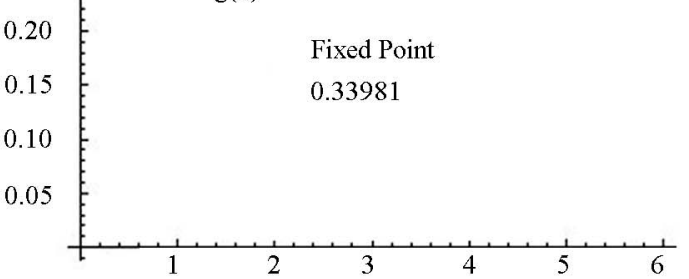

(b)
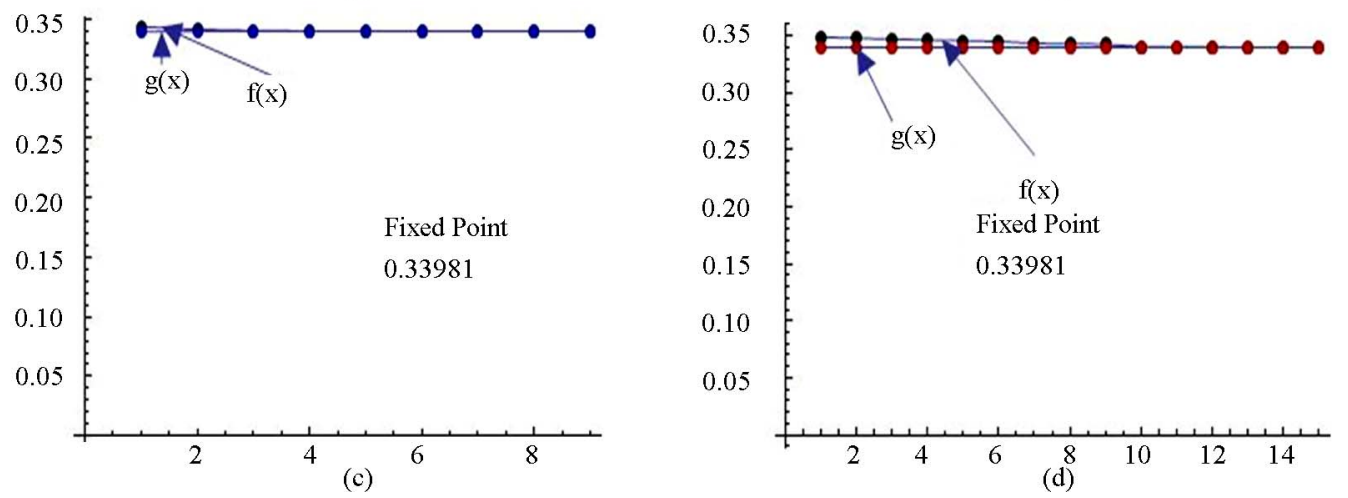

Figure 10. Graphical observations for new modified Ishikawa iteration for $p_{2}(x)$. Here (a)-(d) show the graph for Table 10 . The merging point with value 0.33981 is fixed point. 

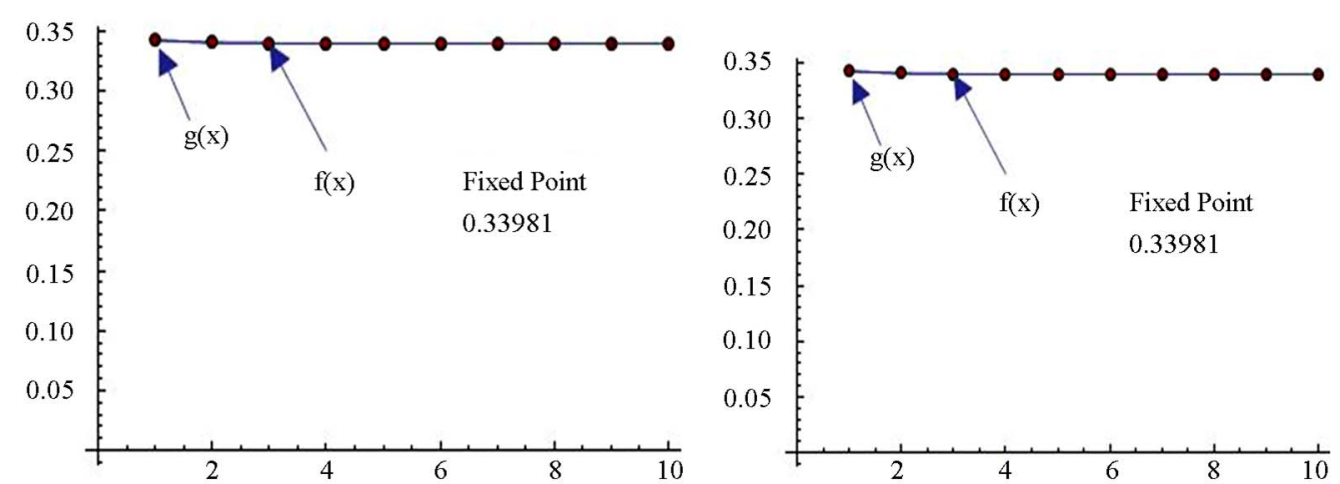

(a)

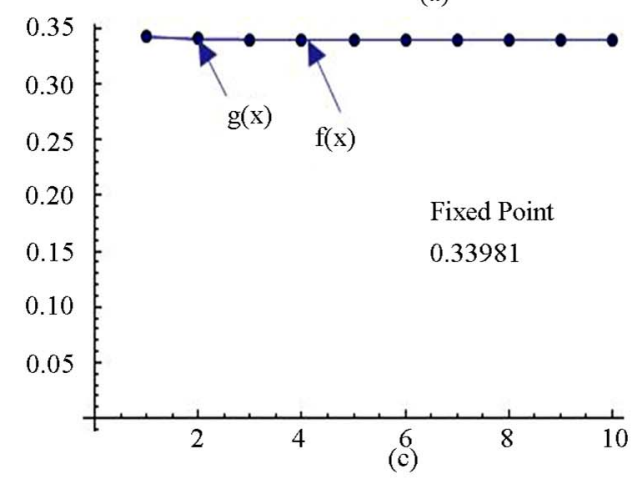

(b)

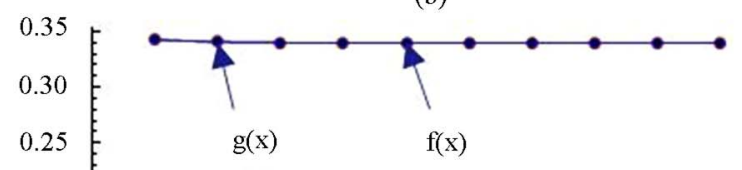

Fixed Point

0.33981

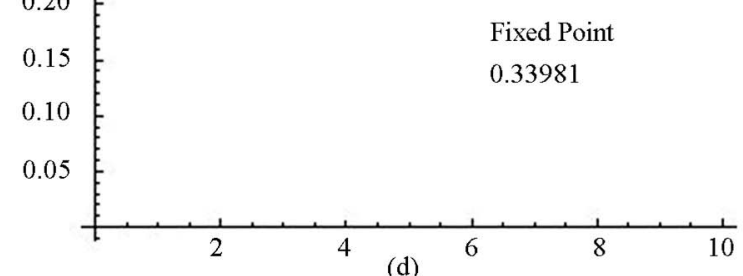

(d)

Figure 11. Graphical observations for simple Agarwal iteration for $p_{2}(x)$. Here (a)-(b) show the graph for Table 11. The merging point with value 0.33981 is fixed point.

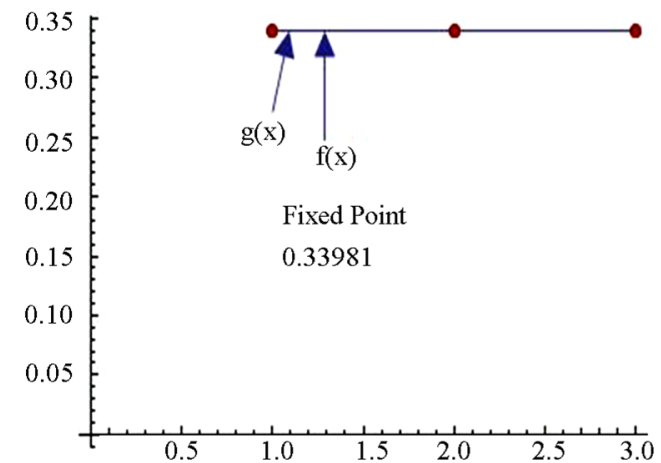

(a)

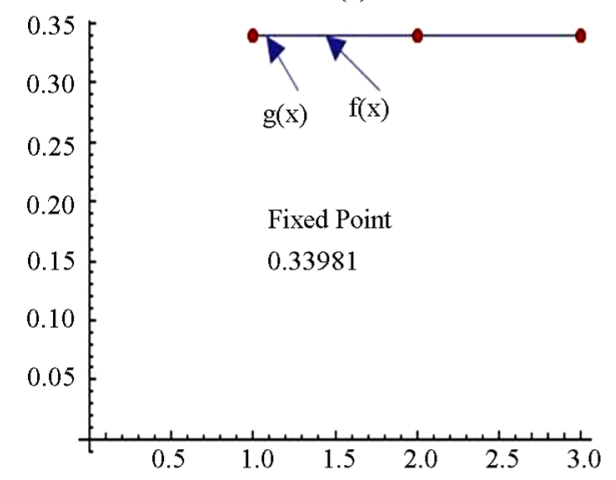

(c)

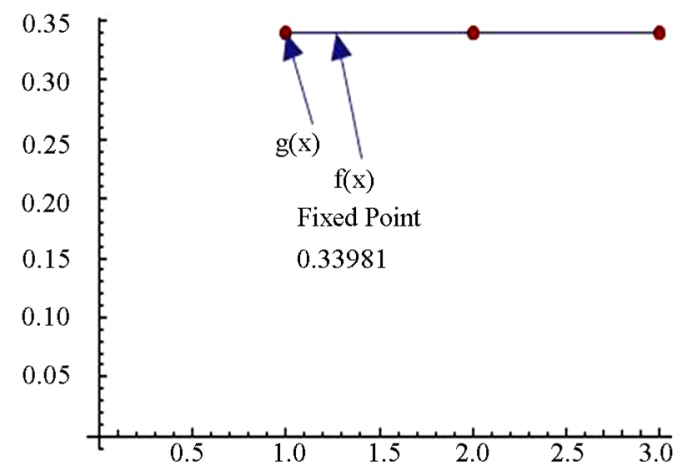

(b)

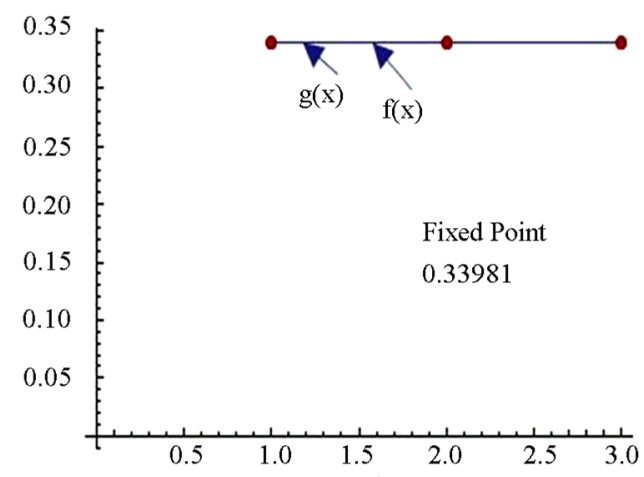

(d)

Figure 12. Graphical observations for simple Agarwal iteration for $p_{2}(x)$. Here (a)-(b) show the graph for Table 12. The merging point with value 0.33981 is fixed point. 


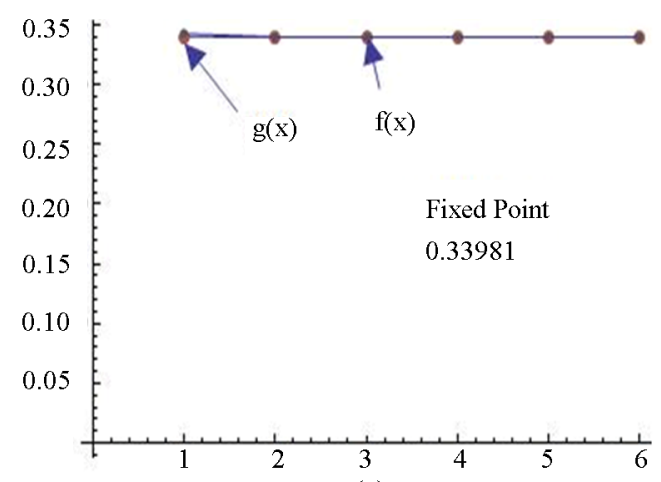

(a)

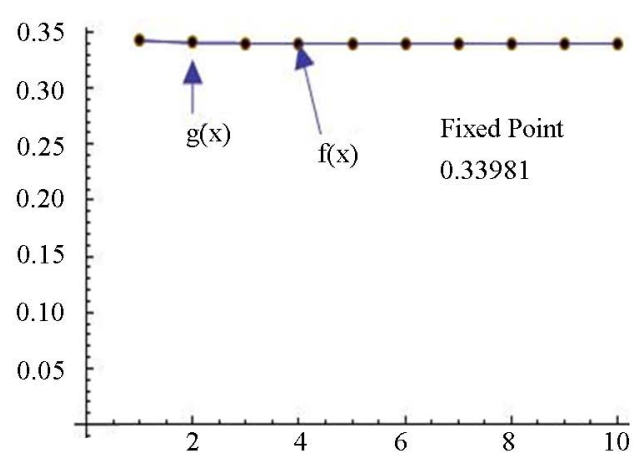

(c)

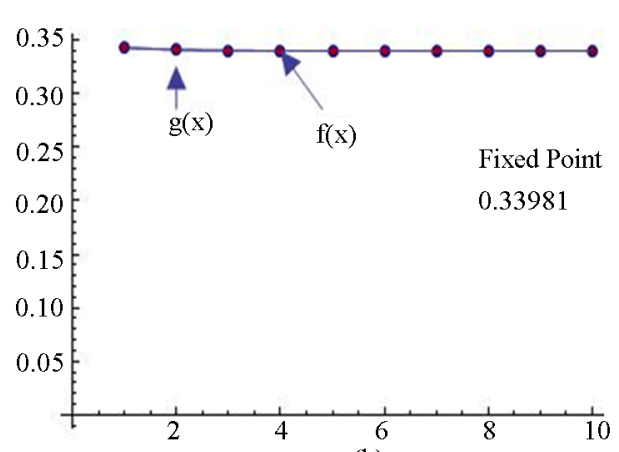

(b)

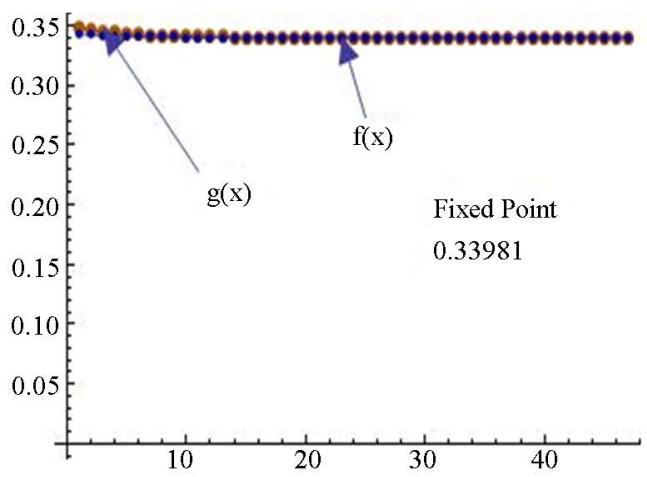

(d)

Figure 13. Graphical observations for simple SP iteration for $p_{2}(x)$. Here (a)-(b) show the graph for Table 13 . The merging point with value 0.33981 is fixed point.
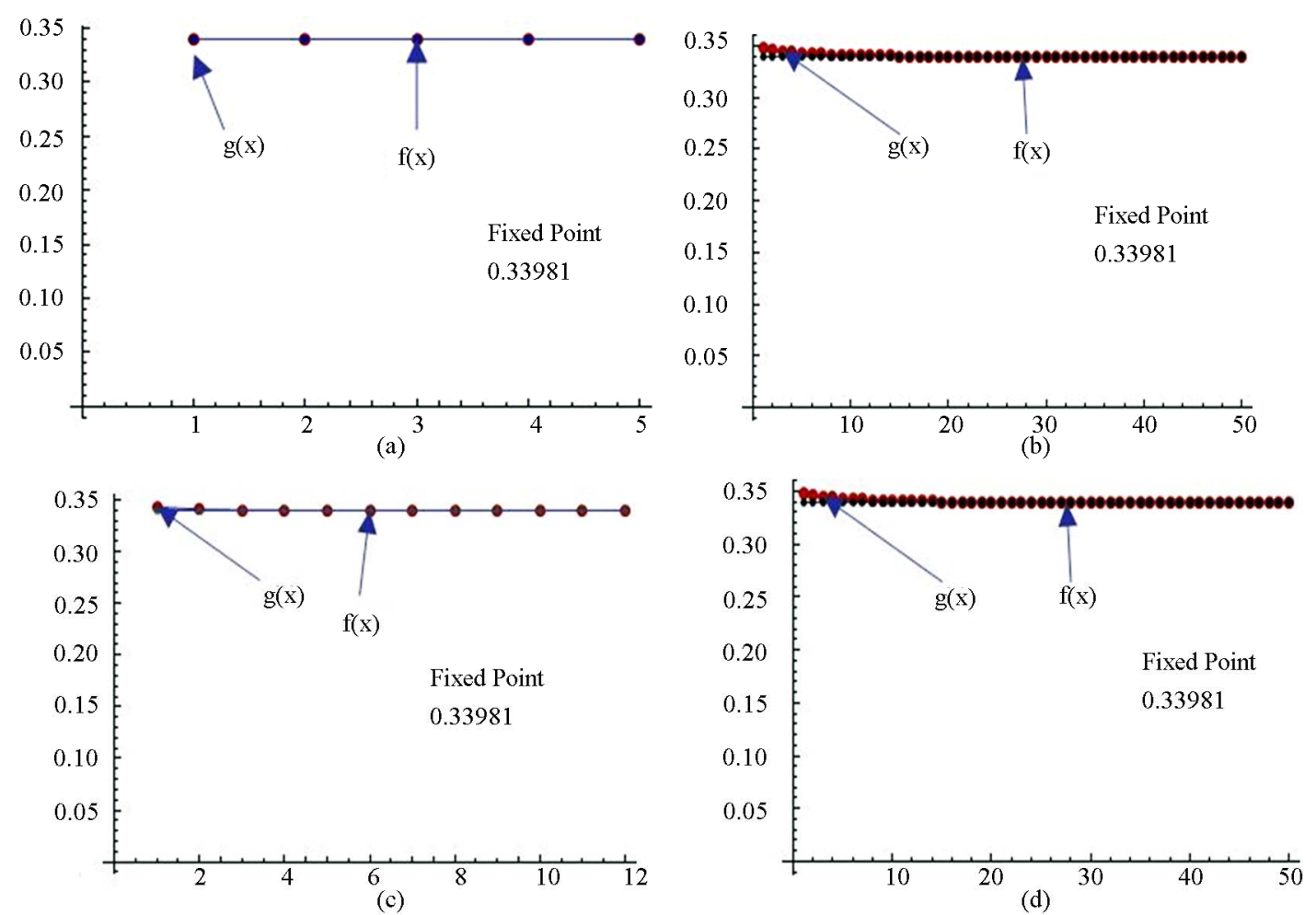

Figure 14. Graphical observations for new modified SP iteration for $p_{2}(x)$. Here (a)-(b) show the graph for Table 14. The merging point with value $\mathbf{0 . 3 3 9 8 1}$ is fixed point. 


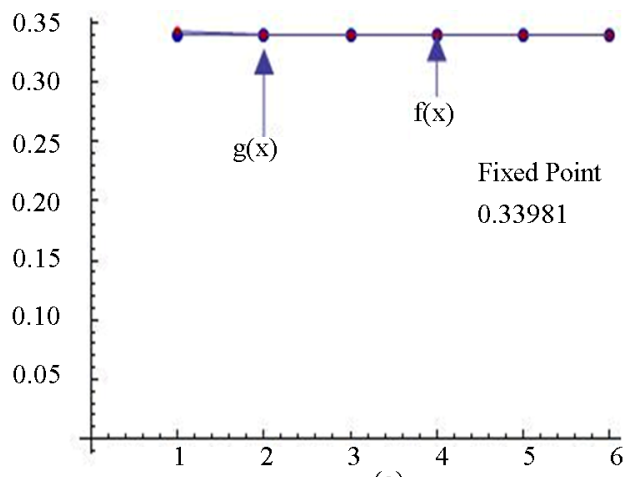

(a)

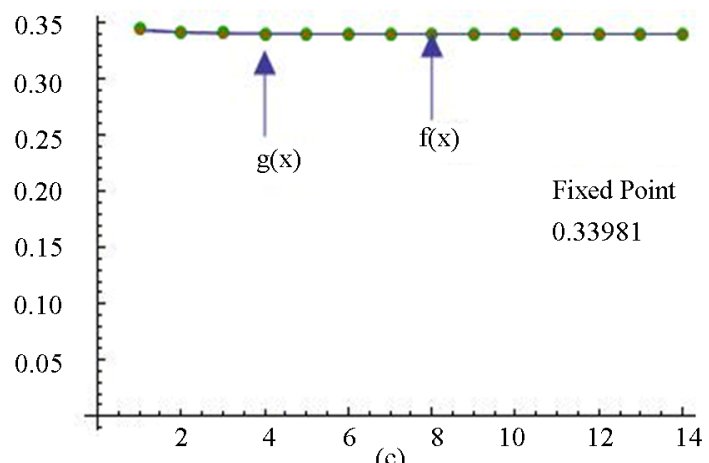

(c)

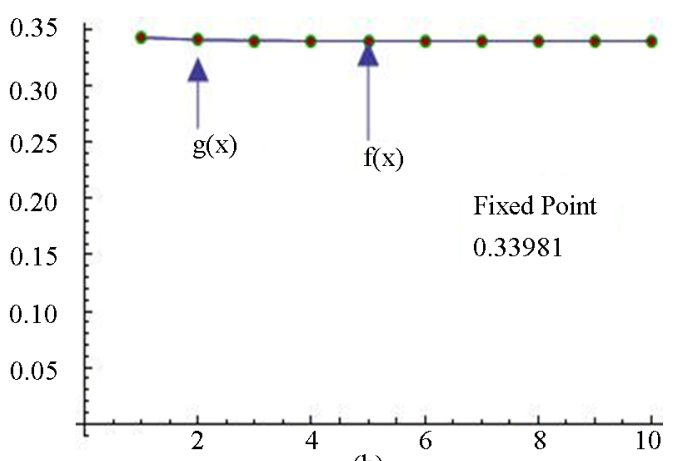

(b)

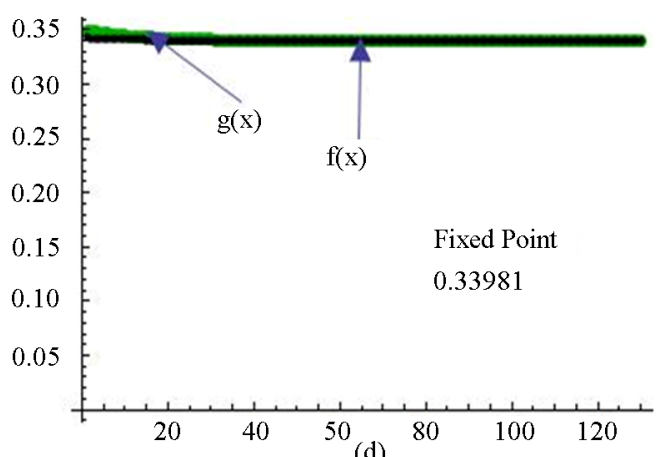

(d)

Figure 15. Graphical observations for simple Noor iteration for $p_{2}(x)$. Here (a)-(b) show the graph for Table 15. The merging point with value 0.33981 is fixed point.

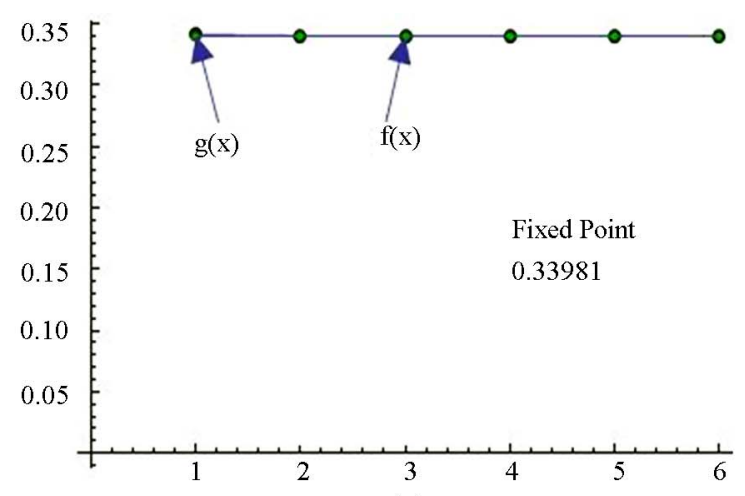

(a)

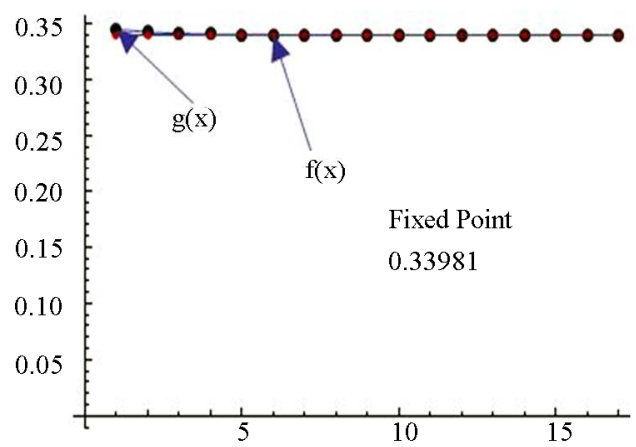

(c)

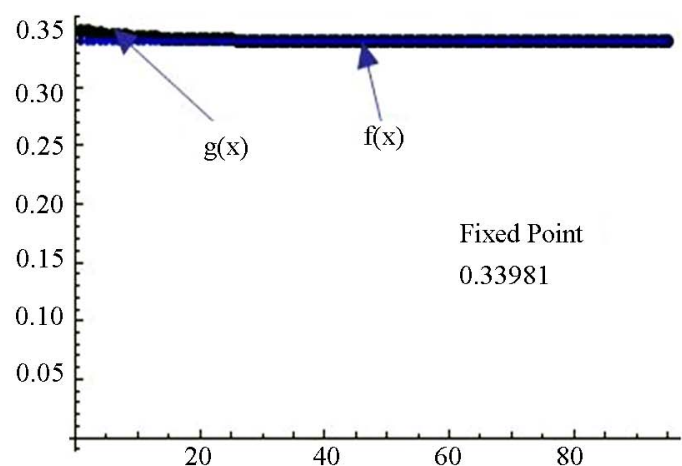

(b)

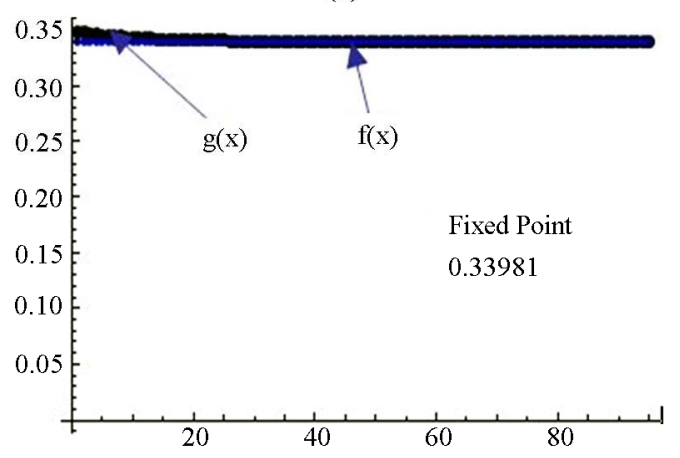

(d)

Figure 16. Graphical observations for new modified Noor iteration for $p_{2}(x)$. Here(a)-(b) show the graph for Table 16. The merging point with value 0.33981 is fixed point. 


\section{Observations}

Table 17. Simple-Ishikawa for $p_{1}(x)$.

\begin{tabular}{ccccccccccccccc}
\hline a/b & 0.001 & 0.01 & 0.1 & 0.2 & 0.3 & 0.4 & 0.5 & 0.6 & 0.7 & 0.8 & 0.9 & 0.99 & 0.999 & 0.9999 \\
0.001 & 5513 & 546 & 49 & 16 & 11 & 6 & 13 & 24 & 86 & not & not & not & not & Not \\
0.01 & 5590 & 554 & 50 & 22 & 12 & 7 & 12 & 24 & 72 & not & not & not & not & not \\
0.1 & 6503 & 645 & 59 & 26 & 15 & 9 & 8 & 12 & 24 & 53 & 3476 & not & not & Not \\
0.2 & 7994 & 794 & 74 & 34 & 20 & 13 & 8 & 6 & 11 & 17 & 28 & 50 & 54 & 54 \\
0.3 & 10455 & 1040 & 99 & 46 & 29 & 20 & 14 & 10 & 7 & 7 & 9 & 13 & 13 & 13 \\
0.4 & 15285 & 1523 & 147 & 70 & 45 & 32 & 24 & 18 & 14 & 12 & 10 & 9 & 9 & 9 \\
0.5 & 29149 & 2909 & 285 & 140 & 91 & 66 & 52 & 42 & 34 & 28 & 21 & 23 & 22 & 22 \\
0.6 & 916911 & 91684 & 9161 & 4577 & 3048 & 2284 & 1826 & 1520 & 1301 & 1137 & 1009 & 916 & 907 & 906 \\
0.7 & not & not & not & not & not & not & not & not & not & not & not & not & not & not \\
0.8 & not & not & not & not & not & not & not & not & not & not & not & not & not & not \\
0.9 & not & not & not & not & not & not & not & not & not & not & not & not & not & not \\
0.99 & not & not & not & not & not & not & not & not & not & not & not & not & not & not \\
0.999 & not & not & not & not & not & not & not & not & not & not & not & not & not & not \\
\hline
\end{tabular}

Table 18. Modified-Ishikawa for $p_{1}(x)$.

\begin{tabular}{|c|c|c|c|c|c|c|c|c|c|c|c|c|c|c|}
\hline $\mathrm{a} / \mathrm{b}$ & 0.001 & 0.01 & 0.1 & 0.2 & 0.3 & 0.4 & 0.5 & 0.6 & 0.7 & 0.8 & 0.9 & 0.99 & 0.999 & 0.9999 \\
\hline 0.001 & 14030 & 1397 & 134 & 63 & 40 & 28 & 21 & 16 & 12 & 9 & 6 & 4 & 4 & 4 \\
\hline 0.01 & 14032 & 1397 & 134 & 63 & 40 & 28 & 21 & 16 & 12 & 9 & 6 & 4 & 4 & 4 \\
\hline 0.1 & 14056 & 1400 & 134 & 63 & 40 & 28 & 21 & 16 & 12 & 9 & 6 & 5 & 4 & 4 \\
\hline 0.2 & 14086 & 1403 & 134 & 64 & 40 & 28 & 21 & 16 & 12 & 9 & 5 & 5 & 4 & 4 \\
\hline 0.3 & 14119 & 1406 & 134 & 64 & 40 & 28 & 21 & 16 & 12 & 9 & 5 & 5 & 4 & 4 \\
\hline 0.4 & 14152 & 1409 & 135 & 64 & 40 & 28 & 21 & 16 & 12 & 9 & 6 & 4 & 4 & 4 \\
\hline 0.5 & 14181 & 1412 & 135 & 64 & 40 & 28 & 21 & 16 & 12 & 9 & 6 & 4 & 4 & 4 \\
\hline 0.6 & 14204 & 1414 & 135 & 64 & 40 & 28 & 21 & 16 & 12 & 9 & 6 & 4 & 4 & 4 \\
\hline 0.7 & 14220 & 1416 & 136 & 64 & 40 & 28 & 21 & 16 & 12 & 9 & 7 & 4 & 4 & 4 \\
\hline 0.8 & 14224 & 1416 & 136 & 64 & 40 & 28 & 21 & 16 & 12 & 9 & 7 & 3 & 3 & 3 \\
\hline 0.9 & 14216 & 1416 & 135 & 64 & 40 & 28 & 21 & 16 & 12 & 9 & 7 & 4 & 3 & 3 \\
\hline 0.99 & 14197 & 1414 & 135 & 64 & 40 & 28 & 21 & 16 & 12 & 9 & 7 & 4 & 3 & 3 \\
\hline 0.999 & 14195 & 1414 & 135 & 64 & 40 & 28 & 21 & 16 & 12 & 9 & 7 & 4 & 3 & 3 \\
\hline
\end{tabular}

Table 19. Simple-Ishikawa for $p_{1}(x)$.

\begin{tabular}{cccccccccccccccccc}
\hline $\mathrm{a} / \mathrm{b}$ & 0.001 & 0.01 & 0.1 & 0.2 & 0.3 & 0.4 & 0.5 & 0.6 & 0.7 & 0.8 & 0.9 & 0.99 & 0.999 & 0.9999 \\
0.001 & 13701 & 1366 & 132 & 65 & 41 & 29 & 23 & 18 & 15 & 12 & 10 & 8 & 8 & 8 & 8 \\
0.01 & 13667 & 1363 & 132 & 64 & 41 & 29 & 22 & 18 & 14 & 12 & 10 & 8 & 8 & 8 \\
0.1 & 13332 & 1329 & 129 & 62 & 40 & 29 & 22 & 17 & 14 & 11 & 9 & 8 & 8 & 8 \\
\hline
\end{tabular}


Continued

\begin{tabular}{llllllllllllllllll}
\hline 0.2 & 12998 & 1296 & 125 & 60 & 39 & 28 & 21 & 17 & 13 & 11 & 9 & 8 & 7 & 7 \\
0.3 & 12671 & 1263 & 122 & 59 & 38 & 27 & 20 & 16 & 13 & 11 & 9 & 7 & 7 & 7 \\
0.4 & 12360 & 1232 & 119 & 57 & 36 & 26 & 20 & 16 & 12 & 10 & 8 & 7 & 7 & 7 \\
0.5 & 12066 & 1203 & 116 & 56 & 36 & 25 & 19 & 15 & 12 & 10 & 8 & 7 & 6 & 6 \\
0.6 & 11785 & 1174 & 113 & 54 & 35 & 25 & 19 & 15 & 12 & 9 & 8 & 6 & 6 & 6 \\
0.7 & 11516 & 1148 & 110 & 53 & 34 & 24 & 18 & 14 & 11 & 9 & 7 & 6 & 6 & 6 \\
0.8 & 11260 & 1122 & 108 & 52 & 33 & 23 & 18 & 14 & 11 & 8 & 7 & 6 & 6 & 5 \\
0.9 & 11015 & 1096 & 106 & 50 & 32 & 23 & 17 & 13 & 10 & 8 & 7 & 5 & 5 & 5 \\
0.99 & 10803 & 1076 & 104 & 49 & 31 & 22 & 17 & 13 & 10 & 8 & 6 & 5 & 5 & 5 \\
0.999 & 10783 & 1074 & 103 & 49 & 31 & 22 & 17 & 13 & 10 & 8 & 6 & 5 & 5 & 5 \\
\hline
\end{tabular}

Table 20. Modified-Ishikawa for $p_{2}(x)$.

\begin{tabular}{cccccccccccccccccc}
\hline $\mathrm{a} / \mathrm{b}$ & 0.001 & 0.01 & 0.1 & 0.2 & 0.3 & 0.4 & 0.5 & 0.6 & 0.7 & 0.8 & 0.9 & 0.99 & 0.999 & 0.9999 \\
0.001 & 9977 & 994 & 95 & 45 & 28 & 20 & 15 & 11 & 9 & 7 & 5 & 3 & 2 & 2 & \\
0.01 & 9977 & 994 & 95 & 45 & 28 & 20 & 15 & 11 & 9 & 7 & 5 & 2 & 2 & 2 \\
0.1 & 9980 & 994 & 95 & 45 & 28 & 20 & 15 & 11 & 9 & 7 & 5 & 2 & 2 & 2 & 2 \\
0.2 & 9983 & 994 & 95 & 45 & 28 & 20 & 15 & 11 & 9 & 7 & 5 & 2 & 2 & 2 \\
0.3 & 9985 & 994 & 95 & 45 & 28 & 20 & 15 & 11 & 9 & 7 & 5 & 2 & 2 & 2 \\
0.4 & 9987 & 995 & 95 & 45 & 29 & 20 & 15 & 11 & 9 & 7 & 5 & 3 & 2 & & 2 \\
0.5 & 9988 & 995 & 95 & 45 & 29 & 20 & 15 & 11 & 9 & 7 & 5 & 3 & 2 & 2 \\
0.6 & 9990 & 995 & 95 & 45 & 29 & 20 & 15 & 11 & 9 & 7 & 5 & 3 & 2 & 2 \\
0.7 & 9991 & 995 & 95 & 45 & 29 & 20 & 15 & 11 & 9 & 7 & 5 & 3 & 2 & 2 \\
0.8 & 9992 & 995 & 95 & 45 & 29 & 20 & 15 & 11 & 9 & 7 & 5 & 3 & 2 & 2 \\
0.9 & 9992 & 995 & 95 & 45 & 29 & 20 & 15 & 11 & 9 & 7 & 5 & 3 & 2 & 2 \\
0.99 & 9992 & 995 & 95 & 45 & 29 & 20 & 15 & 11 & 9 & 7 & 5 & 3 & 2 & 2 \\
0.999 & 9992 & 995 & 95 & 45 & 29 & 20 & 15 & 11 & 9 & 7 & 5 & 3 & 2 & 2 \\
\hline
\end{tabular}

Table 21. Simple-Agarwal for $p_{1}(x)$.

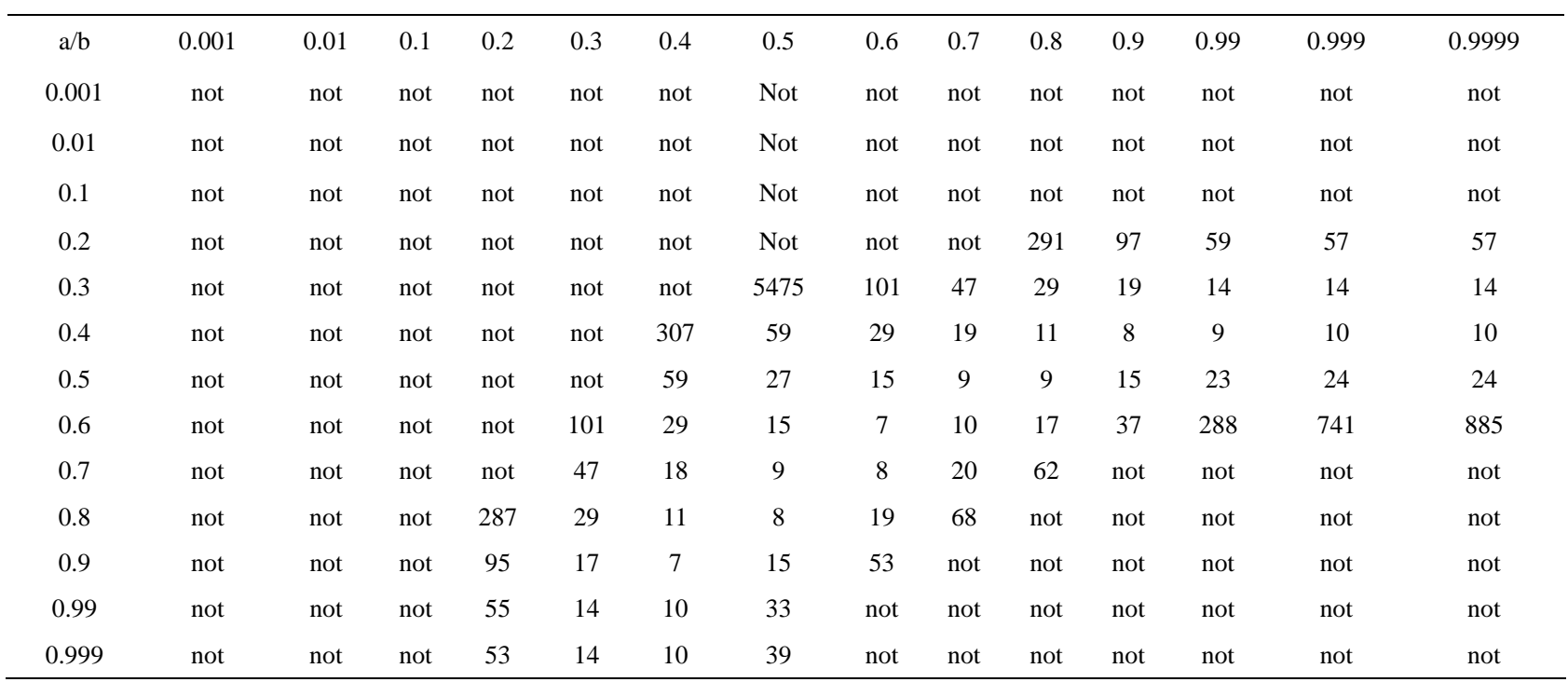


Table 22. Modified-Agarwal for $p_{1}(x)$.

\begin{tabular}{ccccccccccccccccc}
\hline $\mathrm{a} / \mathrm{b}$ & 0.001 & 0.01 & 0.1 & 0.2 & 0.3 & 0.4 & 0.5 & 0.6 & 0.7 & 0.8 & 0.9 & 0.99 & 0.999 & 0.9999 \\
0.001 & 4 & 4 & 4 & 4 & 4 & 4 & 4 & 4 & 4 & 4 & 4 & 4 & 4 & 4 \\
0.01 & 4 & 4 & 4 & 4 & 4 & 4 & 4 & 4 & 4 & 4 & 4 & 4 & 4 & 4 \\
0.1 & 4 & 4 & 4 & 4 & 4 & 4 & 4 & 4 & 4 & 4 & 4 & 4 & 4 & 4 \\
0.2 & 4 & 4 & 4 & 4 & 4 & 4 & 4 & 4 & 4 & 4 & 4 & 4 & 4 & 4 \\
0.3 & 4 & 4 & 4 & 4 & 4 & 4 & 4 & 4 & 4 & 4 & 4 & 4 & 4 & 4 \\
0.4 & 4 & 4 & 4 & 4 & 4 & 4 & 4 & 4 & 4 & 4 & 4 & 4 & 4 & 4 \\
0.5 & 4 & 4 & 4 & 4 & 4 & 4 & 4 & 4 & 4 & 4 & 4 & 4 & 4 & 4 \\
0.6 & 4 & 4 & 4 & 4 & 4 & 4 & 4 & 4 & 4 & 4 & 4 & 4 & 4 & 4 \\
0.7 & 4 & 4 & 4 & 4 & 4 & 4 & 4 & 4 & 4 & 4 & 4 & 4 & 4 & 4 \\
0.8 & 4 & 4 & 4 & 4 & 4 & 4 & 4 & 4 & 4 & 3 & 3 & 3 & 3 & 4 \\
0.9 & 4 & 4 & 4 & 4 & 4 & 4 & 4 & 3 & 3 & 3 & 3 & 3 & 3 & 3 \\
0.99 & 4 & 4 & 4 & 4 & 4 & 4 & 4 & 3 & 3 & 3 & 3 & 3 & 3 & 3 \\
0.999 & 4 & 4 & 4 & 4 & 4 & 4 & 4 & 3 & 3 & 3 & 3 & 3 & 3 & 3 \\
\hline
\end{tabular}

Table 23. Simple-Agarwal for $p_{2}(x)$.

\begin{tabular}{cccccccccccccccc}
\hline $\mathrm{a} / \mathrm{b}$ & 0.001 & 0.01 & 0.1 & 0.2 & 0.3 & 0.4 & 0.5 & 0.6 & 0.7 & 0.8 & 0.9 & 0.99 & 0.999 & 0.9999 \\
0.001 & 8 & 8 & 8 & 8 & 8 & 8 & 8 & 8 & 8 & 8 & 8 & 8 & 8 & 8 \\
0.01 & 8 & 8 & 8 & 8 & 8 & 8 & 8 & 8 & 8 & 8 & 8 & 8 & 8 & 8 \\
0.1 & 8 & 8 & 8 & 8 & 8 & 8 & 8 & 8 & 8 & 8 & 8 & 8 & 8 & 8 \\
0.2 & 8 & 8 & 8 & 8 & 8 & 8 & 8 & 8 & 8 & 8 & 8 & 8 & 7 & 7 & 7 \\
0.3 & 8 & 8 & 8 & 8 & 8 & 8 & 8 & 8 & 7 & 7 & 7 & 7 & 7 & 7 \\
0.4 & 8 & 8 & 8 & 8 & 8 & 8 & 7 & 7 & 7 & 7 & 7 & 7 & 7 & 7 & 6 \\
0.5 & 8 & 8 & 8 & 8 & 8 & 7 & 7 & 7 & 7 & 7 & 7 & 6 & 6 & 6 \\
0.6 & 8 & 8 & 8 & 8 & 8 & 7 & 7 & 7 & 7 & 7 & 6 & 6 & 6 & 6 \\
0.7 & 8 & 8 & 8 & 8 & 7 & 7 & 7 & 7 & 6 & 6 & 6 & 6 & 6 & 6 \\
0.8 & 8 & 8 & 8 & 8 & 7 & 7 & 7 & 7 & 6 & 6 & 6 & 6 & 5 & 5 \\
0.9 & 8 & 8 & 8 & 8 & 7 & 7 & 7 & 6 & 6 & 6 & 5 & 5 & 5 & 5 \\
0.99 & 8 & 8 & 8 & 7 & 7 & 7 & 6 & 6 & 6 & 6 & 5 & 5 & 5 & 5 \\
0.999 & 8 & 8 & 8 & 7 & 7 & 7 & 6 & 6 & 6 & 5 & 5 & 5 & 5 & 5 \\
\hline
\end{tabular}

Table 24. Modified-Agarwal for $p_{2}(x)$.

\begin{tabular}{ccccccccccccccccc}
\hline $\mathrm{a} / \mathrm{b}$ & 0.001 & 0.01 & 0.1 & 0.2 & 0.3 & 0.4 & 0.5 & 0.6 & 0.7 & 0.8 & 0.9 & 0.99 & 0.999 & 0.9999 \\
0.001 & 2 & 2 & 2 & 2 & 2 & 2 & 2 & 2 & 2 & 2 & 2 & 2 & 2 & 2 \\
0.01 & 2 & 2 & 2 & 2 & 2 & 2 & 2 & 2 & 2 & 2 & 2 & 2 & 2 & 2 \\
0.1 & 2 & 2 & 2 & 2 & 2 & 2 & 2 & 2 & 2 & 2 & 2 & 2 & 2 & 2 \\
0.2 & 2 & 2 & 2 & 2 & 2 & 2 & 2 & 2 & 2 & 2 & 2 & 2 & 2 & 2 \\
0.3 & 2 & 2 & 2 & 2 & 2 & 2 & 2 & 2 & 2 & 2 & 2 & 2 & 2 & 2 & 2 \\
0.4 & 2 & 2 & 2 & 2 & 2 & 2 & 2 & 2 & 2 & 2 & 2 & 2 & 2 & & 2 \\
0.5 & 2 & 2 & 2 & 2 & 2 & 2 & 2 & 2 & 2 & 2 & 2 & 2 & 2 & 2 \\
0.6 & 2 & 2 & 2 & 2 & 2 & 2 & 2 & 2 & 2 & 2 & 2 & 2 & 2 & 2 \\
\hline
\end{tabular}


Continued

\begin{tabular}{|c|c|c|c|c|c|c|c|c|c|c|c|c|c|c|}
\hline 0.7 & 2 & 2 & 2 & 2 & 2 & 2 & 2 & 2 & 2 & 2 & 2 & 2 & 2 & 2 \\
\hline 0.8 & 2 & 2 & 2 & 2 & 2 & 2 & 2 & 2 & 2 & 2 & 2 & 2 & 2 & 2 \\
\hline 0.9 & 2 & 2 & 2 & 2 & 2 & 2 & 2 & 2 & 2 & 2 & 2 & 2 & 2 & 2 \\
\hline 0.99 & 2 & 2 & 2 & 2 & 2 & 2 & 2 & 2 & 2 & 2 & 2 & 2 & 2 & 2 \\
\hline 0.999 & 2 & 2 & 2 & 2 & 2 & 2 & 2 & 2 & 2 & 2 & 2 & 2 & 2 & 2 \\
\hline
\end{tabular}

We have noted the converging step of different iterations in tabular form and compare the conversing step for different value of a, b, c. Now by comparative analysis we noted that

1) For $p_{1}(x)$, simple Ishikawa do not converge for $0<a \leq 0.1,0.8<b \leq 1$ and $0.6<a<1,0<b<1$ but new modified Ishikawa converges for all values of a and b converges faster than Ishikawa iteration for corresponding values of a, b. Also it converges at lesser step as a and b both approaches one but not so in case of simple Ishikawa as observe from Tables $\mathbf{1 7}$ and 18. Similarly if we compare the both iterations for $p_{2}(x)$ as observed from Tables 19 and $\mathbf{2 0}$ that as we increase values of a and $\mathrm{b}$ simultaneously than converging step decreases for both iterations but modified Ishikawa iteration converges at lesser step for $p_{2}(x)$.

2) As observed from Tables 21 and 22 for $p_{1}(x)$ simple Agarwal et al. do not converge for all values of a and $\mathrm{b}$ it converges for

$$
\begin{aligned}
& \{a=0.2,0.8 \leq b<1\}, \quad\{a=0.3,0.5 \leq b<1\}, \\
& \{a=0.4,0.5,0.4 \leq b<1\},\{a=0.6,0.3 \leq b<1\}, \\
& \{a=0.7,0.3 \leq b \leq 0.8\}, \quad\{0.8 \leq a<1,0.2 \leq b \leq 0.5\}
\end{aligned}
$$

but modified new Agarwal et al. iteration converges at lesser step for all values of a, b. For $p_{2}(x)$ both iterations converge for all values of $\mathrm{a}$ and $\mathrm{b}$ but modified iteration converges faster than simple iteration

3) The simple SP iteration converges at lesser step for $p_{1}(x)$ when $a=1 / 2, b=1 / 2, c=1 / 2$ as we increases a and $b$ the step of convergence increases. But do not converge if $\mathrm{a}$ and $\mathrm{b}$ approaches to one whereas modified new SP iteration converges for all values of a, b, c and at lesser step than simple SP. For $p_{2}(x)$ both iteration converges for all values of $\mathrm{a}, \mathrm{b}$ and $\mathrm{c}$ but modified new SP converge faster than Simple SP iteration.

4) For simple Noor and modified new Noor iteration result is same as for SP and modified new SP iterations.

\section{Conclusion}

By the observation formed from the program and graph drawn in $\mathrm{C}^{++}$and Mathematica for $p_{1}(x)$ and $p_{2}(x)$ polynomial, we conclude that the modified Ishikawa, Agarwal et al., SP, Noor are faster than simple Ishikawa, Agarwal et al., SP, Noor; but if we compare modified Ishikawa, Agarwal et al., SP, Noor with decreasing order of rate of convergence of modified Agarwal et al., SP, Noor, Ishikawa, modified new Agarwal et al. have consistent rate of convergence. The graphs drawn are based on data formed from $\mathrm{C}^{++}$program and plot the data in mathematica to show the fixed point.

\section{REFERENCES}

[1] J. Biazar and A. Amirteimoori, "An Improvement to the Fixed Point Iterative Method," Applied Mathematics and Computation, Vol. 182, No. 1, 2006, pp. 567-571. http://dx.doi.org/10.1016/j.amc.2006.04.019

[2] B. Prasad and R. Sahni, "Convergence of Some General Iterative Schemes,” International Journal of Mathematical Analysis, Vol. 5, No. 25, 2011, pp. 1237-1242.

[3] S. Ishikawa, "Fixed Points by a New Iteration Method," Proceedings of the American Mathematical Society, Vol. 44, No. 1, 1974, pp. 147-150.

http://dx.doi.org/10.1090/S0002-9939-1974-0336469-5

[4] R. P. Agarwal, D. O’Regan and D. R. Sahu, "Iterative Construction of Fixed Points of Nearly Asymptotically Non-Expansive Mappings," Journal of Nonlinear and Convex Analysis, Vol. 8, No. 1, 2007, pp. 61-79.

[5] M. A. Noor, "New Approximation Schemes for General Variational Inequalities,” Journal of Mathematical Analysis and Applications, Vol. 251, No. 1, 2000, pp. 217-229. http://dx.doi.org/10.1006/jmaa.2000.7042

[6] W. Phuengrattana and S. Suantai, "On the Rate of Convergence of Mann, Ishikawa, Noor and SP Iterations for Continuous Functions on an Arbitrary Interval,” Journal of Computational and Applied Mathematics, Vol. 235, No. 9, 2011, pp. 3006-3014. http://dx.doi.org/10.1016/j.cam.2010.12.022

[7] E. Babolian and J. Biazar, "On the Order of Convergence of Adomian Method," Applied Mathematics and Computation, Vol. 130, No. 2-3, 2002, pp. 383-387. http://dx.doi.org/10.1016/S0096-3003(01)00103-5 